\title{
THE $\Pi_{2}^{1}$-SINGLETON CONJECTURE
}

\author{
SY D. FRIEDMAN
}

\section{INTRODUCTION}

$L$, the universe of constructible sets, was introduced by Gödel to establish the relative consistency of the Axiom of Choice and Continuum Hypothesis with the usual axioms for set theory. The class of constructible sets forms the smallest inner model, by which we mean a subuniverse of the universe of all sets that contains all ordinal numbers and in which the axioms for set theory are true. This minimality for $L$ is a consequence of the fact that constructible sets are totally absolute, in that they each can be defined from ordinal numbers via a definition that is independent of the inner model in which this definition is interpreted.

Cohen produced nonconstructible sets using the method of forcing. However it is in the nature of this method to produce "generic" sets that cannot be explicitly defined. Let us say that a set $x$ is absolute if it can be defined from ordinal numbers via a definition that defines $x$ in every inner model containing $x$. We are particularly interested in the case where $x$ is a set of nonnegative integers, what we call a real. Thus we may ask: is there an absolute, nonconstructible real?

Silver and Solovay produced such a real using large cardinal theory. If there is a measurable cardinal then the uncountable cardinal numbers $\omega_{1}, \omega_{2}, \ldots$ form a nonconstructible sequence. By listing sentences $\phi_{1}, \phi_{2}, \ldots$ in the language of set theory (augmented by names for $\omega_{1}, \omega_{2}, \ldots$ ) they obtain the absolute, nonconstructible real $0^{\sharp}=\left\{n \mid \phi_{n}\right.$ is true in $\left.L\right\}$.

Is $0^{\sharp}$ the "least" such example? If $x, y$ are sets then $x$ is constructible from $y$ if $x$ belongs to $L(y)$, the smallest inner model containing $y$. Our question then becomes: is $0^{\sharp}$ constructible from every absolute, nonconstructible real?

As no real generic in the sense of Cohen can be absolute, a positive answer to our question would follow from the statement that $0^{\sharp}$ is constructible from every real which is not Cohen-generic. However Jensen refuted this last statement by discovering a new kind of generic real. This key result opened up the possibility of obtaining a negative answer to our question. The strongest possible

Received by the editors November 11, 1988; the results of this paper were presented at the European summer meeting of the Association for Symbolic Logic in Padova, Italy, August 1988.

1980 Mathematics Subject Classification (1985 Revision). Primary 03E45, 03E55, 03E15, 03 D60. Research supported by NSF Grant \#8903380-DMS. 
negative answer would consist of a nonconstructible $\Pi_{2}^{1}$-singleton from which $0^{\sharp}$ is not constructible. Solovay's $\Pi_{2}^{1}$-Singleton Conjecture asserts the existence of such a real.

In this paper we construct a Jensen-generic $\Pi_{2}^{1}$-singleton, thereby establishing the $\Pi_{2}^{1}$-Singleton Conjecture.

Theorem. There is a $\Pi_{2}^{1}$-singleton $R, \quad 0<_{L} R<_{L} 0^{\#}$.

We construct an $L$-definable partial order $\mathscr{P}$ such that there is a unique $\mathscr{P}$-generic real, and that real belongs to $L\left[0^{\#}\right]$. Using David's trick (see [D]) we can express this uniqueness in a $\Pi_{2}^{1}$ way.

A generic for $\mathscr{P}$ consists of a real $R$ and a class-sized predicate $A$ such that

(1) $R$ codes $A$, Jensen style (see [BJW]); and

(2) $A$ (together with $0^{\#}$ ) "guarantees" that $R$ is the unique $\mathscr{P}$-generic.

Moreover, such a pair $(R, A)$ is definable in $L\left[0^{\#}\right]$. Indeed:

(3) There is a total $\Sigma_{1}(L)$ "procedure" $\left(i_{1}, \ldots, i_{n}\right) \longmapsto p\left(i_{1}, \ldots, i_{n}\right)$ such that the generic determined by $(R, A)$ is $\left\{p \mid p\left(i_{1}, \ldots, i_{n}\right), p\right.$ are compatible in $\mathscr{P}$ for all $i_{1}<\cdots<i_{n}$ in $\left.I\right\}$, where $I=$ the Silver Indiscernibles for $L$.

The construction of $\mathscr{P}$ requires a $\Sigma_{1}$ index for the function described in (3). Such an index is obtained via the Recursion Theorem.

To construct $\mathscr{P}$ we use both Forward Easton (Jensen Coding) and Backward Easton methods. The latter is needed to create the predicate $A$. Property (2) is achieved as follows: Think of $\left(i_{1}, \ldots, i_{n}\right)$ as a "guess" at an $n$-tuple of Silver indiscernibles. We develop a method of "killing" such guesses (by generically adding certain CUB sets); no correct guess $\left(i_{1}, \ldots, i_{n}\right) \in I^{n}$ can be killed when $i_{1}$ is regular. $A$ is designed to kill any guess $\left(i_{1}, \ldots, i_{n}\right)$ that produces via (3) a condition $p\left(i_{1}, \ldots, i_{n}\right)$ incompatible with our generic real $R$. Thus there is a unique $\mathscr{P}$-generic since any other would lead us to killing correct guesses $\left(i_{1}, \ldots, i_{n}\right) \in I^{n}$ for regular $i_{1}$.

How to KILL A GUESS $\left(i_{1}, \ldots, i_{n}\right)$

As the procedure $\left(i_{1}, \ldots, i_{n}\right) \longmapsto p\left(i_{1}, \ldots, i_{n}\right)$ is $\Sigma_{1}(L)$ we will see that killing $\left(i_{1}, \ldots, i_{n}\right)$ is equivalent to killing $\left(\bar{i}_{1}, \ldots, \bar{i}_{n}\right)$, the image of $\left(i_{1}, \ldots\right.$, $i_{n}$ ) under the transitive collapse of an elementary submodel of $L$ containing $i_{1} \cup\left\{i_{1}, \ldots, i_{n}\right\}$. Thus we will only kill sequences $\left(i_{1}, \ldots, i_{n}\right) \in L_{i_{1}^{+}}$where $i_{1}^{+}$ denotes $\left(i_{1}^{+}\right)^{L}$. (The sequence $\left(\bar{i}_{1}, \ldots, \bar{i}_{n}\right)$ can be thought of as a guess at the collapse of an $n$-tuple of indiscernibles.)

Conventions. For $X \subseteq \mathrm{ORD}, X^{n}=$ all increasing $n$-tuples from $X$. And $\alpha^{+}$ denotes $\left(\alpha^{+}\right)^{L}$ for all $\alpha$. 
Fix $i_{1}<\cdots<i_{n+1}, n \geq 1$ and define $I\left(i_{1}, \ldots, i_{n+1}\right)=\left\{i<i_{1} \mid i\right.$ is $L$ inaccessible and $i, i_{1}$ satisfy the same $\Sigma_{1}$ properties in $L_{i_{n+1}}$ with parameters from $\left.i \cup\left\{i_{2}, \ldots, i_{n}\right\}\right\}$. A guess $\left(i_{1}, \ldots, i_{n+1}\right)$ is acceptable if:

(a) $i_{1}$ is $L$-inaccessible and $n \geq 1$; and

(b) $1 \leq k<l \leq n \longrightarrow i_{k} \in I\left(i_{l}, \ldots, i_{n+1}\right)$.

Lemma 1. (a) $n \geq 1$ and $\left(i_{1}, \ldots, i_{n+1}\right) \in I^{n+1} \longrightarrow\left(i_{1}, \ldots, i_{n+1}\right)$ is acceptable.

(b) Suppose $\left(i_{1}, \ldots, i_{n+1}\right)$ is acceptable. Then $\left(j_{1}, \ldots, j_{n}\right) \in I\left(i_{1}, \ldots, i_{n+1}\right)^{n}$ $\longrightarrow\left(j_{1}, \ldots, j_{n}\right)$ and $\left(i_{1}, \ldots, i_{n}\right)$ satisfy the same $\Sigma_{1}$ properties in $L_{i_{n+1}}$, with parameters from $j_{1}$.

(c) For any $\left(i_{1}, \ldots, i_{n+1}\right) \in \mathrm{ORD}^{n+1}$ there exists $\left(i_{1}, \bar{i}_{2}, \ldots, \bar{i}_{n+1}\right) \in$ $\left(i_{1}^{+}\right)^{n+1}$ such that $I\left(i_{1}, \ldots, i_{n+1}\right)=I\left(i_{1}, \bar{i}_{2}, \ldots, \bar{i}_{n+1}\right)$.

Proof. (a) If $\left(j_{1}, \ldots, j_{m}\right),\left(k_{1}, \ldots, k_{m}\right) \in I^{m}, j_{1} \leq k_{1}$ then $\left(j_{1}, \ldots, j_{m}\right)$, $\left(k_{1}, \ldots, k_{m}\right)$ satisfy the same $\Sigma_{1}$ properties in $L_{i}$ with parameters $<j_{1}$, for any $i \in I$ greater than $j_{m} \cup k_{m}$. This does it.

(b) By induction on $n \geq 1$. If $n=1$ this is clear from the definition of $I\left(i_{1}, i_{2}\right)$. Suppose $n>1$ and the property holds for smaller $n$. Note that as $j_{2}, \ldots, j_{n} \in I\left(i_{1}, \ldots, i_{n+1}\right)$ we certainly have $j_{2}, \ldots, j_{n} \in I\left(i_{1}, i_{3}, \ldots, i_{n+1}\right)$ and hence since $i_{1} \in I\left(i_{2}, i_{3}, \ldots, i_{n+1}\right)$ we get $j_{2}, \ldots, j_{n} \in I\left(i_{2}, i_{3}, \ldots, i_{n+1}\right)$. By induction $\left(j_{2}, \ldots, j_{n}\right),\left(i_{2}, \ldots, i_{n}\right)$ satisfy the same $\Sigma_{1}$ properties in $L_{i_{n}}$ with parameters $<j_{2}$ and hence with parameters $\leq j_{1}$. But $\left(j_{1}, i_{2}, \ldots, i_{n}\right)$ and $\left(i_{1}, i_{2}, \ldots, i_{n}\right)$ satisfy the same $\Sigma_{1}$ properties in $L_{i_{n+1}}$ with parameters $<j_{1}$. Putting these last two facts together we get the desired result.

(c) Just let $\left(i_{1}, \bar{i}_{2}, \ldots, \bar{i}_{n+1}\right)$ be the image of $\left(i_{1}, i_{2}, \ldots, i_{n+1}\right)$ under the transitive collapse of an elementary submodel of $L$ of size $i_{1}$ containing $i_{1} \cup$ $\left\{i_{1}, \ldots, i_{n+1}\right\}$.

A condition in $\mathscr{P}$ will be a pair $p=(\bar{s}, q)$ where $\bar{s}$ is a function that assigns a perfect tree $\bar{s}(s) \subseteq 2^{<\omega}$ to each $s \in\left(2^{<\omega}\right)^{<\omega}=$ all finite sequences of finite strings of 0 's and 1's. In this case we write $p_{o}=\bar{s}$. A sequence $s=$ $\left\langle s_{0}, \ldots, s_{n}\right\rangle \in\left(2^{<\omega}\right)^{<\omega}$ is incompatible with $\bar{s}:\left(2^{<\omega}\right)^{<w} \longrightarrow$ Perfect Trees if $s_{0}$ does not lie on $\bar{s}(\varnothing)$ or for some $i<n, s_{i+1}$ does not lie on $\bar{s}\left(\left\langle s_{0}, \ldots, s_{i}\right\rangle\right)$. Otherwise $s$ lies on $\bar{s}$.

Definition. Fix $s \in\left(2^{<\omega}\right)^{<\omega}$. A guess $\left(i_{1}, \ldots, i_{n+1}\right)$ is $s$-bad if $\left(i_{1}, \ldots, i_{n+1}\right)$ is acceptable, $i_{n+1}<i_{1}^{+}, p\left(i_{1}, \ldots, i_{n}\right) \in L_{i_{n+1}}$ and $s$ is incompatible with $p\left(i_{1}, \ldots, i_{n}\right)_{0}$.

Our desired generic real $R$ is defined from $I=$ Silver Indiscernibles as follows: Let $R(\varnothing)=$ unique path through the trees

$$
\left\{p\left(i_{1}, \ldots, i_{n}\right)_{0}(\varnothing) \mid i_{1}, \ldots, i_{n} \in I\right\}
$$


and inductively for

$$
s_{i+1} \subseteq R\left(\left\langle s_{0}, \ldots, s_{i}\right\rangle\right),
$$

$R\left(\left\langle s_{0}, \ldots, s_{i+1}\right\rangle\right)=$ unique path through the trees

$$
\left\{p\left(i_{1}, \ldots, i_{n}\right)_{0}\left(\left\langle s_{0}, \ldots, s_{i+1}\right\rangle\right) \mid i_{1}, \ldots, i_{n} \in I\right\} .
$$

Then

$$
R=\left\{\left\langle s_{0}, \ldots, s_{n}\right\rangle \mid s_{0} \subseteq R(\phi) \text { and for all } i<n, s_{i+1} \subseteq R\left(\left\langle s_{0}, \ldots, s_{i}\right\rangle\right)\right\} .
$$

Remark. It would be simpler and more natural to instead arrange our forcing $\mathscr{P}$ so that conditions are pairs $(s, q)$ where $s \in 2^{<\omega}$ and thus our generic $R$ is just $\cup\left\{p\left(i_{1}, \ldots, i_{n}\right)_{o} \mid i_{1}<\cdots<i_{n}\right.$ in $\left.I\right\}$. However with this simplified approach I do not see how to carry out the construction of a $\mathscr{P}$-generic from $0^{\#}$.

By Lemma 1 (a), (b) we see that if $\left(i_{1}, \ldots, i_{m+1}\right)$ is $s$-bad where $s=$ $p\left(j_{1}, \ldots, j_{m}\right)_{o},\left(j_{1}, \ldots, j_{m}\right) \in I^{m}$ then $I\left(i_{1}, \ldots, i_{m+1}\right) \cap I$ has cardinality $<m$. Also by Lemma $1(\mathrm{c})$ if $\left(j_{1}, \ldots, j_{m}\right) \in I^{m}$ and $s \in\left(2^{<\omega}\right)^{<\omega}$ is incompatible with $p\left(j_{1}, \ldots, j_{m}\right)_{0}$ then there exists $\left(\bar{j}_{2}, \ldots, \bar{j}_{m+1}\right)$ such that $\left(j_{1}, \bar{j}_{2}, \ldots, \bar{j}_{m+1}\right)$ is $s$-bad and $I\left(j_{1}, \bar{j}_{2}, \ldots, \bar{j}_{m+1}\right) \supseteq I \cap j_{1}$.

In order to kill an acceptable guess $\left(i_{1}, \ldots, i_{n+1}\right)$ we add a CUB to $i_{1}$ $I\left(i_{1}, \ldots, i_{n+1}\right)$. The forcing $\mathbb{Q}(s), s \in\left(2^{<\omega}\right)^{<\omega}$, kills all $s$-bad guesses $\left(i_{1}, \ldots\right.$, $\left.i_{n+1}\right)$ : Form the Backward Easton iteration $\left\langle\mathbb{Q}^{\alpha}(s)\right| \alpha \in$ ORD $\rangle$ as follows. $\mathbb{Q}^{o}(s)=\{\phi\}$ and $\mathbb{Q}^{\alpha+1}(s)=\mathbb{Q}^{\alpha}(s) *\{\phi\}$ unless $\alpha$ is $L$-inaccessible. In the latter event $\mathbb{Q}^{\alpha+1}(s)=\mathbb{Q}^{\alpha}(s) * \mathbb{R}^{\alpha}$ where $\mathbb{Q}^{\alpha}(s) \Vdash \mathbb{R}^{\alpha}$ is the forcing which adds a CUB subset to $\alpha-I\left(\alpha, i_{2}, \ldots, i_{n+1}\right)$ for $s$-bad $\left(\alpha, i_{2}, \ldots, i_{n+1}\right)$, with $<\alpha$-support. (Thus a condition in $\mathbb{R}^{\alpha}$ specifies a bounded closed subset of $\alpha-I\left(\alpha, i_{2}, \ldots, i_{n+1}\right)$ for each of $<\alpha$-many $s$-bad $\left(\alpha, i_{2}, \ldots, i_{n+1}\right)$.) For limit $\lambda, \mathbb{Q}^{\lambda}(s)=$ Direct Limit $\left\langle\mathbb{Q}^{\alpha}(s) \mid \alpha<\lambda\right\rangle$ for regular $\lambda$ and $\mathbb{Q}^{\lambda}(s)=$ Inverse Limit $\left\langle\mathbb{Q}^{\alpha}(s) \mid \alpha<\lambda\right\rangle$ for singular $\lambda$ (there are natural embeddings $\mathbb{Q}^{\alpha}(s) \longrightarrow \mathbb{Q}^{\beta}(s)$ for $\alpha \leq \beta)$. And $\mathbb{Q}(s)=$ Direct Limit $\left\langle\mathbb{Q}^{\alpha}(s) \mid \alpha \in \mathrm{ORD}\right\rangle$.

Remark. A simpler way to kill $s$-bad guesses would be to define $\mathbb{R}^{\alpha}$ so as to add a CUB to $\alpha-\bigcup\left\{I\left(\alpha, i_{2}, \ldots, i_{n_{1}}\right) \mid\left(\alpha, i_{2}, \ldots, i_{n+1}\right) s\right.$-bad $\}$. However we need to kill the different $s$-bad $\left(\alpha, i_{2}, \ldots, i_{n+1}\right)$ "independently" for the sake of our later construction of the desired generic real $R$.

For any $\alpha, \mathbb{Q}(s)$ naturally factors as $\mathbb{Q}^{\alpha}(s) * \mathbb{Q}_{\alpha}(s)$.

Lemma 2. (a) For $\alpha$ regular, $\mathbb{Q}^{\alpha+1}(s)$ is $\alpha^{+}-C C$ (antichains are of size $\leq \alpha$ ).

(b) For all $\alpha, \mathbb{Q}^{\alpha+1}(s) \Vdash A$ dense subset of $\mathbb{Q}_{\alpha+1}(s)$ is $\leq \alpha$-closed (decreasing sequences of length $\leq \alpha$ have lower bounds).

(c) $\mathbb{Q}^{\alpha}(s)$ preserves cofinalities, for all $\alpha$.

(d) $\mathbb{Q}^{\alpha}(s) \cap L_{\alpha^{+}}$is dense in $\mathbb{Q}^{\alpha}(s)$, for all $\alpha$. Moreover, for any $\beta<\alpha$ and $p \in \mathbb{Q}^{\alpha}(s), \quad p \mid \beta \in L_{\beta^{+}}$there exists $q \leq p, q \in \mathbb{Q}^{\alpha}(s) \cap L_{\alpha^{+}}$such that $q|\beta=p| \beta$. 
Proof. By simultaneous induction on $\alpha$. (a) It suffices to show that $\mathbb{Q}^{\alpha}(s) \Vdash \mathbb{R}^{\alpha}$ is $\alpha^{+}-C C$ since $\mathbb{Q}^{\alpha+1}(s)=\mathbb{Q}^{\alpha}(s) * \mathbb{R}^{\alpha}$ and by an inductive use of $(\mathrm{d}), \mathbb{Q}^{\alpha}(s)=$ Direct Limit $\left\langle\mathbb{Q}^{\beta}(s) \mid \beta<\alpha\right\rangle$ has a dense subset of size $\alpha$. By $(\mathrm{c}), \mathbb{Q}^{\alpha}(s) \Vdash \alpha$ regular. Now suppose $G^{\alpha}$ is $\mathbb{Q}^{\alpha}(s)$-generic and $L\left[G^{\alpha}\right] \vDash X \subseteq \mathbb{R}^{\alpha}$ is predense (any condition is compatible with an element of $X$.) We can choose $\beta<\alpha^{+}$ of cofinality $\alpha$ such that $L_{\beta} \prec L_{\alpha^{+}}$and $L\left[G^{\alpha}\right] \vDash X \cap L_{\beta}\left[G^{\alpha}\right]$ is predense in $\mathbb{R}^{\alpha} \cap L_{\beta}\left[G^{\alpha}\right]$ But $L\left[G^{\alpha}\right] \vDash p \in \mathbb{R}^{\alpha} \longrightarrow \bar{p} \in L_{\beta}\left[G^{\alpha}\right]$ where $\bar{p}=p \nmid L_{\beta}\left[G^{\alpha}\right]$ since Domain $(p)$ has size $<\alpha$. Thus if $\bar{q} \leq \bar{p}$ in $\mathbb{R}^{\alpha} \cap L_{\beta}\left[G^{\alpha}\right]$ extends an element of $X \cap L_{\beta}\left[G^{\alpha}\right]$, we get $\bar{q} \wedge p \leq p$ in $\mathbb{R}^{\alpha}$ extends an element of $X \cap L_{\beta}\left[G^{\alpha}\right]$. So $X \cap L_{\beta}\left[G^{\alpha}\right]$ is predense in $\mathbb{R}^{\alpha}$, proving the $\alpha^{+}-C C$.

(b) Note that for acceptable $\left(i_{1}, \ldots, i_{n+1}\right), i$ must be $L$-inaccessible for any $i \in I\left(i_{1}, \ldots, i_{n+1}\right)$. The forcing $\mathbb{Q}_{\alpha+1}(s)$ adds CUB sets to $i_{1}-I\left(i_{1}, \ldots, i_{n+1}\right)$ for $s$-bad $i_{1}$ where $i_{1}>\alpha$. So if $D=$ all conditions in $\mathbb{Q}_{\alpha+1}(s)$ such that any CUB set mentioned is forced to contain an ordinal $\geq \alpha$, then $D$ is dense in $\mathbb{Q}_{\alpha+1}(s)$ and $D$ is $\leq \alpha$-closed.

(c) Suppose $\beta<\alpha$ is regular. By an inductive use of (a), we get that $\mathbb{Q}^{\beta+1}(s)$ is $\beta^{+}-C C$ and by $(b), \mathbb{Q}_{\beta+1}(s)$ is $\leq \beta$-distributive (the intersection of $\leq \beta$ many open dense sets is dense). So any ordinal of cofinality $>\beta$ still has cofinality $>\beta$ after forcing with $\mathbb{Q}^{\beta+1}(s) * \mathbb{Q}_{\beta+1}(s)=\mathbb{Q}(s)$ and hence in $\mathbb{Q}^{\alpha}(s)$, since $G \mathbb{Q}(s)$-generic $\longrightarrow G \cap \mathbb{Q}^{\alpha}(s)$ is $\mathbb{Q}^{\alpha}(s)$-generic. If $|\alpha|$ is regular we therefore have that all cofinalities are preserved since by an inductive use of (d), $\mathbb{Q}^{\alpha}(s)$ has a dense subset of size $|\alpha|$.

If $|\alpha|$ is singular then cofinalities $>\alpha^{+}$are preserved by (d) and cofinality $\alpha^{+}$is preserved since otherwise cofinality $>\beta$ would not be preserved for some $\beta<|\alpha|$.

(d) If $\alpha=\beta+1$ with $\beta$ regular then the result follows by an inductive use of (a). If $\alpha$ is regular then the result follows by induction. And if $\alpha$ is singular argue as follows: Let $\lambda=\operatorname{cof}(\alpha)<\alpha$ and factor $\mathbb{Q}^{\alpha}(s)$ as $Q^{\lambda+1}(s) * \mathbb{Q}$. By an inductive use of $(b)$ we know that $\mathbb{Q}^{\lambda+1}(s) \Vdash \mathbb{Q}$ has a dense, $\leq \lambda$-closed subset. Moreover if $\mathscr{D}$ is a name for this dense, $\leq \lambda$-closed subset, we have by (a) for $\alpha=\lambda$ that for any $p$ in $\mathbb{Q}^{\alpha}(s)$ there exists $q \leq p$ such that $q|\lambda+1 \Vdash q|$ $(\lambda, \alpha) \in \mathscr{D}$ and $q\left\lceil\lambda+1=p\left\lceil\lambda+1\right.\right.$. Now given $p \in \mathbb{Q}^{\alpha}(s)$ we can make $\lambda$ many successive extensions $p_{i}$ of $p$ on $(\lambda, \alpha)$ to guarantee that $p_{i} \mid \alpha_{i} \in L_{\alpha_{1}+}$ for $i<\lambda$, where $\left\langle\alpha_{i} \mid i<\lambda\right\rangle$ is cofinal in $\alpha$ and $\lambda<\alpha_{o}$. The resulting extension $q=p_{\lambda}$ belongs to $L_{\alpha^{+}}$, proving the density of $\mathbb{Q}^{\alpha}(s) \cap L_{\alpha^{+}}$.

So we get that $\mathbb{Q}(s)$ is (cardinal and) cofinality-preserving, by the argument in (c) of the lemma. Note that a $\mathbb{Q}(s)$-generic cannot exist if $s$ is incompatible with some $p\left(i_{1}, \ldots, i_{n}\right)_{0},\left(i_{1}, \ldots, i_{n}\right) \in I^{n}$, for that would lead to a CUB subset of $\omega_{1}$ (in the real world) disjoint from $I \cap \omega_{1}$. This last property is the source for the uniqueness of our $\mathscr{P}$-generic real $R$. 


\section{THE FORCINGS $\mathscr{P}(s)$}

Let $G(s)$ denote a generic for $\mathbb{Q}(s)$. Then $\hat{\mathscr{P}}(s)$ is the iteration $\mathbb{Q}(s) * \hat{\mathbb{R}}^{s}$ where $\hat{\mathbb{R}}^{s}$ is the Jensen coding of $G(s)$ by a subset of $\omega_{1}^{L}$ (see [BJW]). We want to refine $\hat{\mathbb{R}}^{s}$ so that we will get a $\Pi_{2}^{1}$-singleton, via David's trick (see [D]). Thus in Jensen coding $G(s)$ modify the definition of $S_{\alpha}, \alpha \geq \omega: t \in S_{\alpha}$ if $t:[\alpha,|t|) \longrightarrow 2,|t|<\alpha^{+}$, and $\forall \xi \leq|t| \forall \eta, \quad L_{\eta}[t \mid \xi, G(s) \uparrow \alpha] \vDash\left(Z F_{10}+\xi=\right.$ $\alpha^{+} \longrightarrow$ Each $s$-bad guess $\left(i_{1}, \ldots, i_{n+1}\right)$ has been killed), where $Z F_{10}=Z F$ but with only $\Sigma_{10}$-replacement. (We actually could work with full $Z F$ here.) Then $\mathbb{R}^{s}$ denotes the modified Jensen coding of $G(s)$ into $\omega_{1}^{L}$ where $S_{\alpha}$ is defined in this way and $\mathscr{P}(s)=\mathbb{Q}(s) * \mathbb{R}^{s}$. David's arguments show that $\mathscr{P}(s)$ is cofinality-preserving. We sketch a proof here.

Lemma 3. (a) $\mathbb{Q}(s) \Vdash \mathbb{R}^{s}$ preserves cofinalities, and hence $\mathscr{P}(s)$ preserves cofinalities .

(b) If $X(s) \subseteq \omega_{1}$ is $\mathscr{P}(s)$-generic then $X(s)$ codes $G(s)$ and $\forall \xi \forall \eta$, $L_{\eta}[X(s) \cap \xi] \vDash\left(Z F_{10}+\xi=\omega_{1} \longrightarrow\right.$ Each $s$-bad guess has been killed $)$.

Proof sketch. (a) We use notation as in [BJW]. The key is to show that $\mathscr{P}_{\tau}^{s}$ is $\leq \tau$-distributive where $\tau \in \operatorname{Card} \cap \kappa, s \in S_{\kappa}$. Suppose $\kappa=\tau^{+}$so we are dealing with the successor coding $R^{s}$ whose conditions are pairs $(t, \bar{t})$ for almost disjointly coding $s$, with $t \in S_{\tau}$. We can think of $R^{s}$ as a 2-step iteration where first we code $s$ by $D \subseteq \kappa=\tau^{+}$without the restriction that $D \mid \xi \in S_{\tau}$, $\xi<\tau^{+}$and then we add $E \subseteq \tau^{+}$so that $(D \vee E) \mid \xi \in S_{\tau}$ for $\xi<\tau^{+}$. The former forcing is $\leq \tau$-closed. Then note that $\forall \eta \geq \tau^{+}, L_{\eta}[D] \vDash\left(Z F_{10} \longrightarrow\right.$ Each $s$-bad guess has been killed). But then if $L_{-}[D \cap \xi] \stackrel{\Sigma_{\omega}}{\longrightarrow} L_{\eta}[D]$ where $\tau<\xi$ we get that $L_{\bar{\xi}}[D \cap \xi] \vDash\left(Z F_{10} \longrightarrow\right.$ Each $s$-bad guess has been killed). Now consider the forcing to add $E$, via initial segments $u$ such that $u \vee(D \uparrow|u|) \in S_{\tau}$. Extendibility is easy. If we are given a $\tau$-sequence $\left\langle\Delta_{i} \mid i<\tau\right\rangle$ of predense sets to meet then successively make extensions $u_{o} \subseteq u_{1} \subseteq, \cdots$ in the least possible way but guaranteeing that $\left|u_{i}\right|$ is the $\tau^{+}$of the collapse of an elementary submodel of $L[D]$ containing $\tau \cup\left\{\left\langle\Delta_{i} \mid i<\tau\right\rangle\right\}$. The net effect is that $D|| u_{\lambda} \mid \in S_{\tau}$ for limit $\lambda$, so $u_{\lambda}$ is a condition.

Now for $\kappa$ not of the form $\delta^{+}, \delta$ limit we can get the distributivity of $\mathscr{P}_{\tau}^{s}$ by factoring, using the above and induction. We must show that $\mathscr{P}_{\tau}^{s}$ is $\leq \tau$-distributive when $s \in S_{\gamma^{+}}, \quad \gamma$ limit, $\tau<\gamma$. Follow the proof of [BJW], where conditions $p^{0} \geq p^{1} \geq \cdots$ are successively chosen to meet the $\Delta_{i}$ 's in a $\tau$-sequence $\left\langle\Delta_{i} \mid i<\tau\right\rangle$ of predense sets and in addition arrange that for limit $\lambda, p_{\delta}^{\lambda}$ is generic over an appropriate collapsed hull $M_{\delta}^{\lambda}$ for $\delta \in \operatorname{Card} \cap[\tau, \gamma)$. But the genericity of $p_{\delta}^{\lambda}$ guarantees that $p_{\delta}^{\lambda} \in S_{\delta}$, since $p_{\delta}^{\lambda}$ codes conditions in the collapse of the various $S_{\delta^{\prime}}$.

(b) If $\xi<\omega_{1}$ then this is guaranteed by the definition of $S_{\omega}$. If $\xi=\omega_{1}$ then 
this holds by reflection to a countable elementary submodel of $L_{\eta}[X(s)]$.

Next we want to present some properties of $\mathscr{P}(s)=\mathbb{Q}(s) * \mathbb{R}^{s}$ which do not take advantage of the fact that this forcing factors as an iteration. This approach will generalize to a later context where such a factoring is not available.

If $\Delta$ is a set of conditions, $q$ a condition then $q$ meets $\Delta$ if $q \leq r$ for some $r \in \Delta$. If $G$ is a set of conditions then $G$ meets $\Delta$ if some $q \in G$ meets $\Delta$.

Definition. $\Delta \subseteq \mathscr{P}(s)$ is predense on the $\mathbb{Q}(s)$-component if $p=\left(p^{0}, p^{1}\right) \in$ $\mathscr{P}(s) \longrightarrow \exists q \leq p, q$ meets $\Delta$, and $q^{1}=p^{1}$. Suppose $G \subseteq \mathscr{P}(s)$. Then $G$ is $\mathscr{P}(s)$-generic on the $\mathbb{Q}(s)$-component if whenever $\Delta \subseteq \mathscr{P}(s)$ is predense on the $\mathbb{Q}(s)$-component then $G$ meets $\Delta$.

Lemma 4. Suppose $G$ is $\mathscr{P}(s)$-generic. Then $G$ is $\mathscr{P}(s)$-generic on the $\mathbb{Q}(s)$ component.

Proof. Obvious.

Definition. $\Delta \subseteq P(s)$ is predense on the $\mathbb{R}^{s}$-component if $p \in \mathscr{P}(s) \longrightarrow \exists q \leq$ $p, q$ meets $\Delta, q^{0}=p^{0} . G \subseteq \mathscr{P}(s)$ is $\mathscr{P}(s)$-generic on the $\mathbb{R}^{s}$-component if whenever $\Delta \subseteq \mathscr{P}(s)$ is predense on the $\mathbb{R}^{s}$-component then $G$ meets $\Delta$.

Lemma 5. Suppose $G$ is $\mathscr{P}(s)$-generic. Then is $\mathscr{P}(s)$-generic on the $\mathbb{R}^{s}$-component.

Proof. Obvious.

Lemmas 4 and 5 have a converse.

Lemma 6. Suppose $G \subseteq \mathscr{P}(s)$ is compatible $(p, q \in G \longrightarrow \exists r \in G, r \leq p, q)$ and closed upwards $(p \in G, p \leq q \longrightarrow q \in G)$. Suppose $G$ is $\mathscr{P}(s)$-generic on both the $\mathbb{Q}(s)$-component and the $\mathbb{R}^{s}$-component. Then $G$ is $\mathscr{P}(s)$-generic.

Proof. Suppose $\Delta \subseteq \mathscr{P}(s)$ is predense; we want to show that some $q \in G$ meets $\Delta$. Consider $\Delta^{1}=\left\{p \mid \mathbb{Q}(s) \Vdash\left(q^{0}, p^{1}\right)\right.$ meets $\Delta$ for some $\left.q^{0} \in G(s)\right\}$. We claim that $\Delta^{1}$ is predense on the $\mathbb{R}^{s}$-component. Indeed, suppose $p \in \mathscr{P}(s)$ and let $q^{1}=$ least $q^{1} \leq p^{1}$ such that $\left(q^{0}, q^{1}\right)$ meets $\Delta$ for some $q^{0} \in G(s)$, if exists, $=p^{1}$ otherwise. Clearly $\left(p^{0}, q^{1}\right) \leq\left(p^{0}, p^{1}\right)=p$. We must show that $\mathbb{Q}(s) \Vdash$ For some $q^{0} \in G(s)$ there is $q^{1}$ such that $\left(q^{0}, q^{1}\right)$ meets $\Delta$ and $q^{1} \leq p^{1}$ in $\mathbb{R}^{s}$. But this is equivalent to: $\left\{q^{0} \in \mathbb{Q}(s) \mid \exists q^{1}\left(q^{0}, q^{1}\right) \leq\left(q^{0}, p^{1}\right)\right.$ meets $\left.\Delta\right\}$ is dense, and the latter follows from the predensity of $\Delta$. So $\Delta^{1}$ is predense on the $\mathbb{R}^{s}$-component.

Choose $p=\left(p^{0}, p^{1}\right) \in G \cap \Delta^{1}$. Thus $\mathbb{Q}(s) \Vdash\left(q^{0}, p^{1}\right)$ meets $\Delta$ for some $q^{0} \in$ $G(s)$. Now consider $\Delta^{0}=\left\{q \mid\left(q^{0}, p^{1}\right)\right.$ meets $\left.\Delta\right\}$. We claim that $\Delta^{0}$ is predense on the $\mathbb{Q}(s)$-component. Indeed, given $\left(q^{0}, q^{1}\right)$ we can choose $r^{0} \leq q^{0}$ so that $\left(r^{0}, p^{1}\right)$ meets $\Delta$, since this property of $r^{0}$ is dense in $\mathbb{Q}(s)$. Then $\left(r^{0}, q^{1}\right) \leq$ $\left(q^{0}, q^{1}\right)$ belongs to $\Delta^{0}$. As $G$ is $\mathscr{P}(s)$-generic on the $\mathbb{Q}(s)$-component, we can choose $q \leq p$ in $G \cap \Delta^{0}$. But then $q$ meets $\Delta$.

Now we consider the reduction of predense sets, a weakening of genericity. 
Definition. Suppose $p \in \mathscr{P}(s)$ and $\Delta \subseteq \mathscr{P}(s)$ is predense. Then $p$ reduces $\Delta$ below $\gamma$ on the $\mathbb{Q}(s)$-component if $\forall q \leq p \exists r \leq q$ ( $r$ meets $\Delta, r^{1}=q^{1}$ and $\left.r^{0} \uparrow(\gamma, \infty)=q^{0} \uparrow(\gamma, \infty)\right)$. And $p$ reduces $\Delta$ below $\gamma$ on the $\mathbb{R}^{s}$-component if $\forall q \leq p \exists r \leq q\left(r\right.$ meets $\Delta, r^{0}=q^{0}$, and $\mathbb{Q}(s) \Vdash r^{1} \uparrow\left(\gamma^{\prime}, \infty\right)=q^{1} \uparrow\left(\gamma^{\prime}, \infty\right)$ for some $\left.\gamma^{\prime}<\gamma\right)$.

Lemma 7. Suppose $p \in \mathscr{P}(s)$ and $\left\langle\Delta_{i} \mid i<\alpha\right\rangle$ are predense on the $\mathbb{Q}(s)$-component $\left(\mathbb{R}^{s}\right.$-component, respectively), $\alpha$ a regular $L$-cardinal. Then there exists $q \leq p$ such that $q$ reduces each $\Delta_{i}$ below $\alpha$ on the $\mathbb{Q}(s)$-component $\left(\mathbb{R}^{s}\right.$ component, respectively).

Proof. First consider reduction on the $\mathbb{Q}(s)$-component. By Lemma $2, \mathbb{Q}^{\alpha+1}(s)$ is $\alpha^{+}-C C$ and $\mathbb{Q}^{\alpha+1}(s) \Vdash \mathbb{Q}_{\alpha+1}(s)$ is $\leq \alpha$-distributive. And for each $i<\alpha$, $\Delta_{i}^{*}=\left\{q^{0} \in \mathbb{Q}(s) \mid\left(q^{0}, \phi\right)\right.$ meets $\left.\Delta_{i}\right\}$ is dense on $\mathbb{Q}(s)$. Using the above properties of $\mathbb{Q}^{\alpha+1}(s), \mathbb{Q}_{\alpha+1}(s)$ we can now easily extend $p$ to $q$ reducing each $\Delta_{i}$ below $\alpha$ on the $\mathbb{Q}(s)$-component.

Now consider reduction on the $\mathbb{R}^{s}$-component. If $\alpha=\omega$ then the result holds by $\leq \omega$-distributivity of $\mathbb{R}^{s}$. If $\alpha$ is a successor $L$-cardinal then the result holds by the $\leq \alpha$-distributivity of $\mathbb{R}_{\alpha}^{s}$ (the coding of $G(s)$ into $\alpha^{+}$) and the $\alpha^{+}-C C$ of the coding into $\alpha$. For inaccessible $\alpha$ the result follows from $\Delta$-distributivity: if $\Delta_{i}$ is predense on $\mathbb{R}_{i^{+}}^{s}$ for $i<\alpha$ then $\left\{r \in \mathbb{R}^{s} \mid(r)_{i^{+}}\right.$meets $\Delta_{i}$ for all $\left.i<\alpha\right\}$ is dense on $\mathbb{R}^{s}$. (See [BJW].)

We generalize the above to $\prod_{s} \mathscr{P}(s)$. It will be necessary to deal with the equivalent forcing $\mathscr{P}^{*}=\left(\prod_{s} \mathbb{Q}(s)\right) *\left(\prod_{s} \mathbb{R}^{s}\right)=$ all pairs $\left(p^{0}, p^{1}\right)$ where $p^{0} \in$ $\prod_{s} \mathbb{Q}(s)=\mathbb{Q}$ and $p^{1}$ is a $\mathbb{Q}$-term, $\mathbb{Q} \Vdash \operatorname{Dom}\left(p^{1}\right)=\left(2^{<\omega}\right)^{<\omega}$ and $\forall s\left(p^{1}(s) \in\right.$ $L[G(s)]$ is a condition in the Jensen coding of $G(s)$. Note that $\mathbb{Q}$ is $\leq \omega$ distributive so a dense subset of $\mathbb{Q} *\left(\prod_{s} \mathbb{R}^{s}\right)$ consists of $\left(p^{0}, p^{1}\right)$ where $p^{0} \in \mathbb{Q}$, for some constructible $f:\left(2^{<\omega}\right)^{<\omega} \longrightarrow$ Terms, $\forall s\left(\mathbb{Q} \Vdash p^{1}(s)=f(s)\right.$ and $f(s)$ is a $\mathbb{Q}(s)$-term). Thus $\prod_{s} \mathscr{P}(s)$ is isomorphic to a dense subset of $\mathscr{P}^{*}=$ $\mathbb{Q} * \prod_{s} \mathbb{R}^{s}=\mathbb{Q} * \mathbb{R}$.

Definitions. $\Delta \subseteq \mathscr{P}^{*}$ is predense on the $\mathbb{Q}$-component ( $\mathbb{R}$-component, respectively) if $p \in \mathscr{P}^{*} \longrightarrow \exists q \leq p, q$ meets $\Delta$, and $q^{1}=p^{1}\left(q^{0}=p^{0}\right.$, respectively). $G \subseteq \mathscr{P}^{*}$ is $\mathscr{P}^{*}$-generic on the $\mathbb{Q}$-component if whenever $\Delta \subseteq \mathscr{P}^{*}$ is predense on the $\mathbb{Q}$-component, then $G$ meets $\Delta . G \subseteq \mathscr{P}^{*}$ is $\mathscr{P}^{*}$-generic on the $\mathbb{R}$ component if whenever $\Delta \subseteq \mathscr{P}^{*}$ is predense on the $\mathbb{R}$-component, then $G$ meets $\Delta$.

Lemma 8. (a) Suppose $K$ is $\mathscr{P}^{*}$-generic. Then $K$ is $\mathscr{P}^{*}$-generic on the $\mathbb{Q}$ component and on the $\mathbb{R}$-component.

(b) Conversely, if $K \subseteq \mathscr{P}^{*}$ is $\mathscr{P}^{*}$-generic on the $\mathbb{Q}, \mathbb{R}$-components and $K$ is compatible, closed upwards then $K$ is $\mathscr{P}^{*}$-generic.

Proof. Exactly as in Lemmas 4, 5, 6.

We also consider the reduction of predense sets for $\mathscr{P}^{*}$. 
Definitions. Suppose $p \in \mathscr{P}^{*}$ and $\Delta \subseteq \mathscr{P}^{*}$ is predense. Then $p$ reduces $\Delta$ below $\gamma$ on the $\mathbb{Q}$-component if $\forall q \leq p \exists r \leq q$ ( $r$ meets $\Delta, r^{1}=q^{1}$, and $r^{0}(s) \uparrow(\gamma, \infty)=q^{0}(s) \uparrow(\gamma, \infty)$ for all $\left.s\right)$. And $p$ reduces $\Delta$ below $\gamma$ on the $\mathbb{R}$-component if $\forall q \leq p \exists r \leq q$ (r meets $\Delta, r^{0}=q^{0}$, and $\mathbb{Q} \Vdash$ For some $\gamma^{\prime}<\gamma$, $\left.\forall s r^{1}(s) \uparrow\left(\gamma^{\prime}, \infty\right)=q^{1}(s) \uparrow\left(\gamma^{\prime}, \infty\right)\right)$.

Lemma 9. Suppose $p \in \mathscr{P}^{*}$ and $\left\langle\Delta_{i} \mid i<\alpha\right\rangle$ are predense on the $\mathbb{Q}$-component ( $\mathbb{R}$-component, respectively), $\alpha$ a regular $L$-cardinal. Then there exists $q \leq$ $p$ such that $q$ reduces each $\Delta_{i}$ below $\alpha$ on the $\mathbb{Q}$-component ( $\mathbb{R}$-component, respectively).

Proof. The proof turns on two claims.

Claim 1. $\mathbb{Q} \simeq \mathbb{Q}^{\alpha+1} \times \mathbb{Q}_{\alpha+1}$ where $\mathbb{Q}^{\alpha+1}$ is $\alpha^{+}-C C$ and $\mathbb{Q}^{\alpha+1} \Vdash \mathbb{Q}_{\alpha+1}$ is $\leq \alpha$-distributive.

As $\mathbb{Q}=\prod_{s} \mathbb{Q}(s)=\prod_{s} \mathbb{Q}^{\alpha+1}(s) * \mathbb{Q}_{\alpha+1}(s)$ we take

$$
\begin{aligned}
\mathbb{Q}^{\alpha+1}= & \prod_{s} \mathbb{Q}^{\alpha+1}(s), \mathbb{Q}_{\alpha+1}=\prod_{s} \mathbb{Q}_{\alpha+1}(s) \\
= & \left\{f \in L\left[G^{\alpha+1}\right] \mid \text { Domain }(f)=\left(2^{<\omega}\right)^{<\omega}\right. \text { and } \\
& \left.\forall s\left(f(s) \in \mathbb{Q}_{\alpha+1}(s) \subseteq L\left[G^{\alpha+1}(s)\right]\right)\right\} .
\end{aligned}
$$

Thus $\mathbb{Q}=\left\{\left(p^{0}, p^{1}\right) \mid p^{0} \in \mathbb{Q}\right.$ and $p^{1}$ is a $\mathbb{Q}$-term, $\mathbb{Q} \Vdash \operatorname{Dom}\left(p^{1}\right)=\left(2^{<\omega}\right)^{<\omega}$ and $\forall s\left(p^{1}(s)\right.$ is a condition in $\left.\left.\mathbb{Q}_{\alpha+1}(s) \subseteq L\left[G^{\alpha+1}(s)\right]\right)\right\}$. Note that in the previous, $p^{1}(s)$ names an element of $L\left[G^{\alpha+1}(s)\right]$, not an arbitrary element of $L\left[G^{\alpha+1}\right]$.

Claim 1 is proved as was Lemma 2, with one new twist: To see that $\mathbb{Q}^{\alpha+1} \Vdash$ $\mathbb{Q}_{\alpha+1}$ is $\leq \alpha$-distributive we must show that if $G^{\alpha+1}$ is $\mathbb{Q}^{\alpha+1}$-generic and $\left\langle\Delta_{i}\right| i<$ $\alpha\rangle$ are predense on $\mathbb{Q}_{\alpha+1}^{G^{n+1}}, \quad\left\langle\Delta_{i} \mid i<\alpha\right\rangle \in L\left[G^{\alpha+1}\right]$ then $\forall p \in \mathbb{Q}_{\alpha+1}^{G^{n+1}} \exists q \leq p(q$ meets each $\Delta_{i}$ ). If we successively extend $p=p_{0} \geq p_{1} \geq \cdots$ to meet the $\Delta_{i}$ 's, there is the danger that for limit $\lambda, p_{\lambda}(s)$ no longer belongs to $L\left[G^{\alpha+1}(s)\right]$, only to $L\left[G^{\alpha+1}\right]$, since the $\left\langle p_{i} \mid i<\lambda\right\rangle$-sequence is defined in $L\left[G^{\alpha+1}\right]$. To overcome this difficulty, as stage $i+1$ instead choose $p_{i+1}$ by first selecting a condition $q_{0}$ in $\mathbb{Q}^{\alpha+1}$ such that for some $p_{i+1}^{q_{0}}, q_{0} \Vdash p_{i+1}^{q_{0}}$ meets $\Delta$, and $\mathbb{Q}^{\alpha+1} \Vdash p_{i+1}^{q_{0}} \leq p_{i}$, then $q_{1}$ incompatible with $q_{0}$ such that for some $p_{i+1}^{q_{1}}$, $q_{1} \Vdash p_{i+1}^{q_{1}}$ meets $\Delta_{i}$ and $\mathbb{Q}^{\alpha+1} \Vdash p_{i+1}^{q_{1}} \leq p_{i+1}^{q_{0}}$, etc. Then after $\beta<\alpha^{+}$stages we get $p_{i+1}=$ greatest lower bound $\left\{p_{i+1}^{q_{j}} \mid j<\beta\right\}$ so that $\mathbb{Q}^{\alpha+1} \Vdash p_{i+1} \leq p_{i}$ and $p_{i+1}(s) \in L\left[G^{\alpha+1}(s)\right]$ for all $s$. (Lemma 2 (b) guarantees that we can arrange that lower bounds exist at limit stages.) This proves the $\leq \alpha$-distributivity of $\mathbb{Q}_{\alpha+1}$. The $\alpha^{+}-C C$ of $\mathbb{Q}^{\alpha+1}$ is as in the proof of Lemma 2, using the fact that conditions in $\mathbb{Q}^{\alpha+1}$ have support size $<\alpha=\alpha^{\aleph_{0}}$. This proves Claim 1.

Claim 2. $\mathbb{Q} \Vdash \mathbb{R} \simeq \mathbb{R}_{\alpha} * \mathbb{R}^{\alpha}$ where $\mathbb{R}_{\alpha}=\left\{p^{1}|[\alpha, \infty)| p^{1} \in \mathbb{R}\right\}$ is $\leq \alpha$ distributive (and $\Delta$-distributive for $L$-inaccessible $\alpha$ ) and $\mathbb{R}^{\alpha}$ is $\alpha^{+}-C C$ for successor $L$-cardinals $\alpha$. 
For the first part it suffices to show that $\mathbb{Q}^{\alpha+1} \Vdash \mathbb{Q}_{\alpha+1} * \mathbb{R}_{\alpha}$ is $\leq \alpha$-distributive. But we can prove this as in Claim 1 , by guessing over an antichain in $\mathbb{Q}^{\alpha+1}$. (Given the $\leq \beta$-distributivity of $\mathbb{R}_{\beta}$ for $\beta<\alpha$ inaccessible, the $\Delta$ distributivity of $\mathbb{R}_{\alpha}$ is easy.) The second part is clear, using $\alpha^{\aleph_{0}}=\alpha$.

Now we are in a position to repeat the proof of Lemma 7.

Corollary 10. $\mathscr{P}^{*}$ preserves cofinalities, and hence so does $\prod_{s} \mathscr{P}(s)$.

\section{THE FORCING $\mathscr{P}$}

Now we use a method for creating(local) $\Pi_{2}^{1}$-singletons (see [JS]). We write a $\prod_{s} \mathscr{P}(s)$-generic as $\prod_{s}\left(G(s), X^{s}\right)$ (where $G(s)$ is $\mathbb{Q}(s)$-generic, $X^{s}$ is a subset of $\omega_{1}^{L}$ coding $G(s)$ ). We introduce a forcing $\mathscr{C}$ over the ground model $L\left[\prod_{s}\left(G(s), X^{s}\right)\right] . \mathscr{C}=\prod_{s} \mathscr{C}(s)$ where $\mathscr{C}(s)$ is a forcing for coding $X^{s}$ by a real using perfect trees.

For $X \subseteq \omega_{1}^{L}$ define ordinals $\mu_{\alpha}(X), \alpha<\omega_{1}^{L}$, inductively as follows:

$$
\bar{\mu}_{0}(X)=\omega, \quad \bar{\mu}_{\alpha}(X)=\bigcup\left\{\mu_{\beta}(X) \mid \beta<\alpha\right\},
$$

$\mu_{\alpha}(X)=$ least $\mu>\bar{\mu}_{\alpha}(X)$ such that $L_{\mu}[X \cap \alpha]$ is admissible and locally countable (that is, $L_{\mu}[X \cap \alpha] \vDash \Sigma_{1}$-replacement and every set is countable). And let $\mathscr{A}_{\alpha}(X)$ denote the structure $L_{\mu_{r}(X)}[X \cap \alpha]$. A real $R$ codes $X$ below $\alpha$ if for all $\beta<\alpha, \beta \in X$ iff $\mathscr{A}_{\beta}(X)[R]$ is admissible.

A perfect tree $T: 2^{<\omega} \longrightarrow 2^{<\omega}$ is such a function satisfying: $T(u * 0), T(u * 1)$ are incompatible extensions of $T(u)$, for all $u \in 2^{<\omega}$. The stem of $T$, denoted stem $(T)$, is $T(\phi)$. A real $R$ is a path through $T, R \in[T]$, if $R=\bigcup\{T(u) \mid u \subseteq$ $S\}$ for some $S \in 2^{\omega}$.

A condition in $\mathscr{C}(s)$ is a perfect tree $T$ such that each path $R$ through $T$ codes $X^{s}$ below $|T|$, where $|T|=$ least $\alpha$ such that $T \in \mathscr{A}_{\alpha}(X)$. Extension is defined by $T_{1} \leq T_{2}$ iff $T_{1}=T_{2} \circ T$ for some tree $T$. Finally $\mathscr{C}=\prod_{s} \mathscr{C}(s)$.

Lemma 11. (a) $T \in \mathscr{C}(s), \alpha<\omega_{1}^{L} \longrightarrow \exists T^{\prime} \leq T,\left|T^{\prime}\right| \geq \alpha$.

(b) $\mathscr{C}$ is a cofinality-preserving forcing.

Proof. (a) By induction on $\alpha$. If $\alpha=\beta+1$ we can assume by induction that $|T|=\beta$ and hence $T \in \mathscr{A}_{\beta}\left(X^{s}\right)$. If $\beta \notin X^{s}$ then we can thin $T$ to $T^{\prime} \in \mathscr{A}_{\beta+1}\left(X^{s}\right)$ so that with the help of $T$, each $R \in\left[T^{\prime}\right]$ codes some real coding $\mu_{\beta}(X)$. If $\beta \in X^{s}$ then we use the fact that $\mu_{\beta}(X)$ is countable in $\mathscr{A}_{\beta+1}(X)$ to thin $T$ to $T^{\prime} \in \mathscr{A}_{\beta+1}(X)$ so that each $R \in\left[T^{\prime}\right]$ is generic over $\mathscr{A}_{\beta}(X)$ for the Cohen-like forcing defined by the nodes of $T$.

For limit $\alpha$, apply induction and the fact that $\bar{\mu}_{\alpha}(X)$ is countable in $\mathscr{A}_{\alpha}(X)$.

(b) Note that $\mathscr{C}(s)$ obeys fusion: if $D_{n} \subseteq \mathscr{C}(s)$ is open and $n$-dense for each $n$ then $\bigcap\left\{D_{n} \mid n<\omega\right\}$ is dense, where $D$ is $n$-dense if $\forall T \exists T^{\prime} \leq T\left(T^{\prime} \in D\right.$ and $T^{\prime}(u)=T(u)$ for $u$ of length $\left.\leq n\right)$. To show that $\mathscr{C}$ preserves cofinalities it 
suffices to observe that $\mathscr{C}$ obeys fusion for the following notion of $n$-dense: $D$ is $n$-dense iff for every $c \in \mathscr{C}$ there is $c^{\prime} \leq c, c^{\prime} \in D$ such that $c^{\prime}\left(s_{k}\right)(u)=$ $c\left(s_{k}\right)(u)$ for $k \leq n$, length $(u) \leq n$ where $\left\langle s_{k} \mid k \in \omega\right\rangle$ enumerates $\left(2^{<\omega}\right)^{<\omega}$.

To show that $\prod_{s} \mathscr{P}(s) * \mathscr{C}(s)$ preserves cofinalities. But this can be established as in Claim 2 of Lemma 9, using the fact that $\mathbb{R}^{s} * \mathscr{C}(s)$ obeys fusion instead of $\leq \omega$-distributivity.

Suppose $\left\langle R(s) \mid s \in\left(2^{<\omega}\right)^{<\omega}\right\rangle$ is $\mathscr{C}$-generic over $L\left[\prod_{s}\left(G(s), X^{s}\right)\right]$. Let $R=$ $\left\{\left\langle s_{0}, \ldots, s_{n}\right\rangle \mid s_{0} \subseteq R(\phi)\right.$ and for all $\left.i<n, s_{i+1} \subseteq R\left(\left\langle s_{0}, \ldots, s_{i}\right\rangle\right)\right\}$. Thus we have:

$$
R \in L\left[\prod_{s}\left(G(s), X^{s}\right)\right]\left[\left\langle R(s) \mid s \in\left(2^{<\omega}\right)^{<\omega}\right\rangle\right]
$$

a generic extension of $L$, preserving cofinalities.

Our desired forcing is $\mathscr{P}$, a forcing that produces the generic extension $L[R]$ of $L$. We will show that a $\mathscr{P}$-generic exists in $L\left[0^{\#}\right.$. But note that no $\prod_{s} \mathscr{P}(s)$ generic can exist (in the presence of $0^{\#}$ ) as that would lead to a CUB subset of $\omega_{1}$ disjoint from $I$. (Indeed no $\mathbb{Q}(s)$-generic can exist for $s$ incompatible with $R$. ) Thus we can think of $L[R] \subseteq L\left[0^{*}\right]$ as a generic extension of $L$, obtained as an inner model of a generic extension $L\left[\prod_{s}\left(G(s), X^{s}\right), \quad\left\langle R(s) \mid s \in\left(2^{<\omega}\right)^{<\omega}\right\rangle\right]$ which "lies outside the real universe."

We give an explicit description of $\mathscr{P}$. A condition is a pair $(\bar{s}, q)$ where $\bar{s}:\left(2^{<\omega}\right)^{<\omega} \longrightarrow$ Perfect Trees, $q \in \prod_{t} \mathscr{P}(t)$ and for each $t, q(t) \Vdash \bar{s}(t) \in$ $\mathscr{C}(t)$. A condition $(\bar{t}, r)$ extends $(\bar{s}, q)$ if whenever $t$ lies on $\bar{t}, \bar{t}(t) \leq \bar{s}(t)$ and $r(t) \leq q(t)$. (We do not require the latter conditions when $t$ is incompatible with $\bar{t}$.) A $\mathscr{P}$-generic $G$ is completely determined by the real $R=\{s \in$ $\left(2^{<\omega}\right)^{<\omega} \mid s$ lies on $\bar{s}$ for all $\left.(\bar{s}, q) \in G\right\}$. Moreover $\left(\prod_{s} \mathscr{P}(s)\right) * \mathscr{C}$-generics yield $\mathscr{P}$-generics, so $\mathscr{P}$ preserves cofinalities:

Lemma 12. Suppose $(K, J)$ is $\prod_{s} \mathscr{P}(s) * \mathscr{C}$-generic. Let $G=\{(\bar{s}, q) \mid$ For some $r \in K, \bar{t} \in J$ we have that $r(s), \bar{t}(s)$ agree with $q(s), \bar{s}(s)$ for $s$ which lie on $\bar{s}$ \}. Then $G$ is $\mathscr{P}$-generic.

Proof. $G$ is clearly compatible and closed upwards. Suppose $\Delta \subseteq \mathscr{P}$ is predense. Consider $\Delta^{*}=\left\{\left(q^{\prime}, \tau\right) \mid\right.$ For some $(\bar{s}, q)$ meeting $\Delta, q^{\prime} \Vdash \tau=\bar{s}$ and $q(s)=q^{\prime}(s)$ for $s$ lying on $\left.\bar{s}\right\}$. Then $\Delta^{*}$ is dense on $\left(\prod_{s} \mathscr{P}(s)\right) * \mathscr{C}$ so $(K, J)$ meets $\Delta^{*}$. So $G$ meets $\Delta$.

Corollary 13. $\mathscr{P}$ preserves cofinalities.

Our treatment of $\mathscr{P}$ is greatly facilitated by embedding it, as we did $\prod_{s} \mathscr{P}(s)$, as a suborder of a larger partial order. Let $\hat{\mathscr{P}}$ consist of all pairs $(\bar{s}, q)$ where $\bar{s}:\left(2^{<\omega}\right)^{<\omega} \longrightarrow$ Perfect Trees, $q=\left(q^{0}, q^{1}\right) \in \mathscr{P}^{*}=\prod_{s} \mathbb{Q}(s) * \prod_{s} \mathbb{R}^{s}$ and for each $s, q^{0} \Vdash\left(q^{1}(s), \bar{s}(s)\right) \in \mathbb{R}^{s} * \mathscr{C}(s)$. And $(\bar{t}, r) \leq(\bar{s}, q)$ in $\hat{\mathscr{P}}$ if for all 
$t$ lying on $\bar{t}, \quad r^{0}(t) \leq q^{0}(t)$ in $\mathbb{Q}(t), \bar{t}(t)$ is a perfect subtree of $\bar{s}(t)$ and $r^{0}[t] \Vdash r^{1}(t) \leq q^{1}(t)$, where $r^{0}[t](s)=\varnothing$ if $s$ is not an initial segment of $t,=r^{0}(s)$ otherwise.

Lemma 14. $\mathscr{P}$ and $\hat{\mathscr{P}}$ are equivalent forcings.

Proof. As in Lemma 12, we have the following: Suppose $\tilde{G}$ is $\Pi_{s} \mathbb{Q}(s) * \Pi_{s} \mathbb{R}^{s} *$ $\mathscr{C}(s)$-generic and write an element of this forcing as $\left(q^{0},\left(q^{1}, \bar{s}\right)\right)$ where $q^{0} \in$ $\prod_{s} \mathbb{Q}(s), \quad q^{0} \Vdash\left(q^{1}(s), \bar{s}(s)\right) \in \mathbb{R}^{s} * \mathscr{C}(s)$ for all $s \in\left(2^{<\omega}\right)^{<\omega}$. Then $\hat{G}$ is $\hat{\mathscr{P}}$. generic where $\hat{G}=\left\{(\bar{s}, q) \mid\right.$ For some $\left(r^{0},\left(r^{1}, \bar{t}\right)\right)$ in $\tilde{G}$ we have that $q^{0}(s)=$ $r^{0}(s), \quad q^{0} \Vdash\left(q^{1}(s), \bar{s}(s)\right)=\left(r^{1}(s), \bar{t}(s)\right)$ for all $s$ lying on $\left.\bar{s}\right\}$.

Now notice that both $\prod_{s} \mathscr{P}(s) * \prod_{s} \mathscr{C}(s)$ and $\prod_{s} \mathbb{Q}(s) * \prod_{s} \mathbb{R}^{s} * \mathscr{C}(s)$ sit densely inside $\prod_{s} \mathbb{Q}(s) * \prod_{s} \mathbb{R}^{s} * \Pi_{s} \mathscr{C}(s)=\mathscr{S}$. If $H$ is $\mathscr{S}$-generic then in $L[H]$ we define therefore a real $R$ which is both $\mathscr{P}$ and $\hat{\mathscr{P}}$ generic. It follows that the $\mathscr{P}$-generic reals are the same as the $\hat{\mathscr{P}}$-generic reals.

Remarks. (1) I do not see how to densely embed $\mathscr{P}$ into $\hat{\mathscr{P}}$ and suspect that no such embedding exists. The difficulty is that in order to specify $q^{1}(t)$ as a $\mathbb{Q}(t)$-term as a constructible function of $t$, we may need to consider more than $q^{0}[t]$.

(2) The necessity of using $\hat{\mathscr{P}}$, where $q^{1}$ is viewed as a $\prod_{s} \mathbb{Q}(s)$-term, is revealed in the proof of Lemma 15.

Our goal is to build a $\mathscr{P}$-generic in $L\left[0^{\#}\right]$. By Lemma 14 it suffices to build a $\hat{\mathscr{P}}$-generic in $L\left[0^{\#}\right]$. The advantage of working with $\hat{\mathscr{P}}$ is that the componentgenericity and predensity reduction techniques that we developed for $\mathscr{P}^{*}$ can be adapted. What we need, are analogues of Lemmas $8(b)$ and 9. First some definitions.

Definitions. For $p \in \hat{\mathscr{P}}$ write $p=(\bar{s}(p), q(p)) . \Delta \subseteq \hat{\mathscr{P}}$ is predense on the $\mathbb{Q}$-component if $p \in \hat{\mathscr{P}} \longrightarrow \exists p_{0} \leq p, p_{0}$ meets $\Delta$ and $q\left(p_{0}\right)^{1}=q(p)^{1} . \Delta \subseteq \mathscr{P}$ is predense on the $\mathbb{R}$-component if $p \in \hat{\mathscr{P}} \longrightarrow \exists p_{0} \leq p, p_{0}$ meets $\Delta$, and $q\left(p_{0}\right)^{0}=q(p)^{0}, \mathbb{Q} \Vdash q\left(p_{0}\right)^{1} \leq q(p)^{1}$.

$K \subseteq \hat{\mathscr{P}}$ is $\hat{\mathscr{P}}$-generic on the $\mathbb{Q}$-component if whenever $\Delta \subseteq \hat{\mathscr{P}}$ is predense on the $\mathbb{Q}$-component, $K$ meets $\Delta . K$ is $\hat{\mathscr{P}}$-generic on the $\mathbb{R}$-component if whenever $\Delta \subseteq \hat{\mathscr{P}}$ is predense on the $\mathbb{R}$-component, $K$ meets $\Delta$.

Lemma 15. Suppose $K \subseteq \hat{\mathscr{P}}$ is compatible, closed upwards, and $\hat{\mathscr{P}}$-generic on the $\mathbb{Q}, \mathbb{R}$-components. Then $K$ is $\hat{\mathscr{P}}$-generic.

Proof. We argue as in the proof of Lemma 6. Suppose $\Delta \subseteq \hat{\mathscr{P}}$ is predense; we want to show that some $p \in K$ meets $\Delta$. For each $\bar{t}$ consider $\Delta^{1}(\bar{t})=\{p \mid \mathbb{Q} \Vdash$ There is $\bar{s} \leq \bar{t}$ such that $\left(\bar{s},\left(q^{0}, q(p)^{1}\right)\right)$ meets $\Delta$ for some $\left.q_{0} \in G\right\}$ where $\mathbb{Q}=\prod_{s} \mathbb{Q}(s), G$ denotes a $\mathbb{Q}$-generic. We claim that $\Delta^{1}(\bar{t})$ is predense on the $\mathbb{R}$-component. Indeed, suppose $p \in \hat{\mathscr{P}}$ and let $q^{1}$ be the $\mathbb{Q}$-term defined by: $q^{1}=$ least $q^{1} \leq q(p)^{1}$ such that for some $\bar{s} \leq \bar{t},\left(\bar{s},\left(q^{0}, q^{1}\right)\right)$ meets $\Delta$ 
for some $q^{0} \in G$, if exists; $q^{1}=q(p)^{1}$ otherwise. Clearly $\mathbb{Q} \Vdash q^{1} \leq q(p)^{1}$. And $\mathbb{Q} \Vdash\left(\right.$ There is $q^{1} \leq q(p)^{1}$ such that $\left(\bar{s},\left(q^{0}, q^{1}\right)\right)$ meets $\Delta$ for some $q^{0} \in G, \bar{s} \leq \bar{t}$ ), as this is a dense property of $q^{0}$ (due to the predensity of $\Delta$ ). So $\Delta^{1}(\bar{t})$ is predense on the $\mathbb{R}$-component.

For each $\bar{t}$ choose $\left(\phi,\left(p^{0}(\bar{t}), p^{1}(\bar{t})\right)\right) \in K$ meeting $\Delta^{1}(\bar{t})$, by the $\hat{\mathscr{P}}_{\text {-generi- }}$ city of $K$ on the $\mathbb{R}$-component. Thus for each $\bar{t}, \mathbb{Q} \Vdash$ There is $\bar{s} \leq \bar{t}$ such that $\left(\bar{s},\left(q^{0}, p^{1}(\bar{t})\right)\right)$ meets $\Delta$ for some $q^{0} \in G$. Now consider $\Delta^{0}(\bar{t})=\{p \mid$ For some $\bar{s} \leq \bar{t},\left(\bar{s},\left(q(p)^{0}, p^{1}(\bar{t})\right)\right)$ meets $\left.\Delta\right\} . \Delta^{0}(\bar{t})$ is predense on the $\mathbb{Q}$ component for each $\bar{t}$. Moreover $\bar{\Delta}^{0}(\bar{t})=\left\{p^{0} \mid p \in \Delta^{0}(\bar{t})\right\}$ is dense open on $\mathbb{Q}$ for each $\bar{t}$ and hence by $\leq \omega_{1}$-distributivity of $\mathbb{Q}, \Delta^{0}=\bigcap\left\{\Delta^{0}(\bar{t}) \mid \bar{t}:\left(2^{<\omega}\right)^{<\omega} \longrightarrow\right.$ Perfect Trees $\}$ is predense on the $\mathbb{Q}$-component. Now choose $\left(\varnothing,\left(r^{0}, r^{1}\right)\right) \in K$ meeting $\Delta^{0}$, by the $\hat{\mathscr{P}}$-genericity of $K$ on the $\mathbb{Q}$-component. Finally let $\Delta_{0}=$ $\left\{p \mid\left(\bar{s}(p),\left(r^{0}, p^{1}(\bar{s}(p))\right)\right)\right.$ meets $\left.\Delta\right\}$. Then $\Delta_{0}$ is predense on the $\mathbb{Q}$-component (and $\mathbb{R}$-component) so we can choose $(\bar{s},(\phi, \phi)) \in K$ meeting $\Delta_{0}$. As $K$ is compatible and closed upwards we get $\left(\bar{s},\left(r^{0}, p^{1}(\bar{s})\right)\right) \in K$ and the latter condition meets $\Delta$.

Remark. The use of $\leq \omega_{1}$-distributivity of $\mathbb{Q}$ can be eliminated if we redefine $\hat{\mathscr{P}}$ so as to allow $\bar{s}$ to be a $\mathbb{Q}$-term.

Definition. Suppose $p \in \hat{\mathscr{P}}$ and $\Delta \subseteq \hat{\mathscr{P}}$ is predense. Then $p$ reduces $\Delta$ below $\gamma$ on the $\mathbb{Q}$-component if $\forall q \leq p \exists r \leq q \quad\left(r^{1}=q^{1}, r\right.$ meets $\Delta, r^{0}(u)$ $(\gamma, \infty)=q^{0}(u) \uparrow(\gamma, \infty)$ for all $\left.u\right)$. And $p$ reduces $\Delta$ below $\gamma$ on the $\mathbb{R}$ component if $\forall q \leq p \exists r \leq q\left(r^{0}=q^{0}, r\right.$ meets $\Delta, \mathbb{Q} \Vdash$ For some $\gamma^{\prime}<\gamma$, $r^{1}(u) \uparrow\left(\gamma^{\prime}, \infty\right)=q^{1}(u) \uparrow\left(\gamma^{\prime}, \infty\right)$ for all $\left.u\right)$.

Lemma 16. Suppose $p \in \hat{\mathscr{P}}$ and $\left\langle\Delta_{i} \mid i<\alpha\right\rangle$ are predense on the $\mathbb{Q}$-component $(\mathbb{R}$-component, respectively), $\alpha$ a regular $L$-cardinal. Then there exists $q \leq p$ such that $q$ reduces each $\Delta_{i}$ below $\alpha$ on the $\mathbb{Q}$-component ( $q$ reduces each $\Delta_{i}$, $i<\beta$ below $\beta$ on the $\mathbb{R}$-component for each $L$-cardinal $\beta \leq \alpha$, respectively).

Proof. As in Lemma 7, using the claims of Lemma 9 (and Fusion for $\mathscr{C}$ when $\alpha=\omega)$.

\section{BUILDING A $\mathscr{P}$-GENERIC FROM $0^{\#}$}

We work with $\hat{\mathscr{P}}$, rather than with $\mathscr{P}$. Thus our goal is to produce a $\Sigma_{1}(L)$ procedure $\left(i_{1}, \ldots, i_{n}\right) \longmapsto p\left(i_{1}, \ldots, i_{n}\right)$ such that $\{p \in \hat{\mathscr{P}} \mid p$ is compatible with $p\left(i_{1}, \ldots, i_{n}\right)$ for all $\left.\left(i_{1}, \ldots, i_{n}\right) \in I^{n}\right\}$ is $\hat{\mathscr{P}}_{\text {-generic. }}$

It is important to observe that the very definition of $\hat{\mathscr{P}}$ made use of an index for this procedure. Thus we are really describing an index for $\left(i_{1}, \ldots, i_{n}\right) \longmapsto$ $p\left(i_{1}, \ldots, i_{n}\right)$ given a "guess" at an index for this procedure. The Recursion Theorem tells us that we can know a proper guess. We must only observe that we are defining here a total procedure, even if our guess at an index for it defines a partial procedure. 
Of course our procedure will produce a $\hat{\mathscr{P}}$-generic from the Silver Indiscernibles I only given a correct guess for it; incorrect guesses would lead us to kill sequences $\left(i_{1}, \ldots, i_{n+1}\right) \in I^{n+1}$ for regular $i_{1}$, an impossibility.

We describe below how to construct a $\hat{\mathscr{P}}$-generic using I (given a correct guess); this construction will easily be made into the desired $\Sigma_{1}(L)$-procedure. The construction is a mixture of Beller's refinement of Jensen's method for obtaining class-generic subsets of $\omega_{1}^{L}$ in $L\left[0^{\#}\right]$ (see [BJW, $\left.\S \S 4.4,5.1\right]$ ), together with a method for obtaining Backward Easton generics in $L\left[0^{\#}\right]$ (see [F1]). In the former case, conditions $p\left(k_{1}, \ldots, k_{n}\right)$ are chosen of domain $L$-Card $\cap\left[\omega, k_{1}^{+}\right]$, where $I=\left\{k_{1}<k_{2}<\cdots\right\}$, and the rest of the generic is determined by indiscernibility preservation: if $p\left(k_{1}, \ldots, k_{n}\right)$ is chosen then the generic has all conditions $p(\vec{k}), \vec{k} \in I^{n}$ (defined from $\vec{k}$ just as was $p\left(k_{1}, \ldots, k_{n}\right)$ from $\left.\left(k_{1}, \ldots, k_{n}\right)\right)$; the entire generic consists of conditions extended by some $p(\vec{k})$, with restraint added at finitely many indiscernibles. In the latter case, conditions $q\left(k_{1}, \ldots, k_{n}\right)$ are chosen successively of length $\leq k_{2}+1$, with the restriction that $G\left(k_{2}\right)$ extend $G\left(k_{1}\right)$. The rest of the generic is determined by indiscernibility preservation, as it consists of all conditions extended by some $q(\vec{k}), \vec{k} \in I^{n}$ (defined from $\vec{k}$ just as was $q\left(k_{1}, \ldots, k_{n}\right.$ ) from $\left.\left(k_{1}, \ldots, k_{n}\right)\right)$. What we do here is to carry out these constructions simultaneously, Jensen's on the $\mathbb{R}$-component and the Backward Easton method on the $\mathbb{Q}$-component. The advantage of working with $\hat{\mathscr{P}}$ is that we can use Lemma 15 to build our $\hat{\mathscr{P}}$-generic by performing the $\mathbb{R}$-component and $\mathbb{Q}$-component constructions independently.

We make one more observation before beginning the construction: Note that the Jensen construction only produces a generic after restraint is added at finitely many indiscernibles. This is the main reason why we write our desired generic as $\left\{p \in \hat{\mathscr{P}} \mid p\right.$ is compatible with each $\left.p\left(i_{1}, \ldots, i_{n}\right),\left(i_{1}, \ldots, i_{n}\right) \in I^{n}\right\}$, rather than $\left\{p \in \hat{\mathscr{P}} \mid p\left(i_{1}, \ldots, i_{n}\right) \leq p\right.$ for some $\left.\left(i_{1}, \ldots, i_{n}\right) \in I^{n}\right\}$.

Here is the construction. Our goal is to produce a $\hat{\mathscr{P}} \cap L_{k_{\omega}}$-generic $K_{0} \subseteq L_{k_{\omega}}$ which is preserved by elementary embeddings $L_{k_{\omega}} \longrightarrow L_{k_{t}}$ obtained by moving indiscernibles $k_{n}, n<\omega$. Thus $K_{0}=\left\{p \in \mathscr{\mathscr { P }} \cap L_{k_{\text {co }}} \mid p\right.$ is compatible with each $\left.p(\vec{k}), \vec{k} \in\left(I \cap k_{\omega}\right)^{<\omega}\right\}$ and the desired $\hat{\mathscr{P}}_{\text {-generic is } K=\{p \in \hat{\mathscr{P}} \mid p}$ is compatible with each $\left.p(\vec{k}), \vec{k} \in I^{<\omega}\right\}$. We define $p_{0} \geq p_{1} \geq p_{2} \geq \cdots$ in $\hat{\mathscr{P}} \cap L_{k_{\omega}}$ by defining $p_{n}=p\left(k_{1}, \ldots, k_{n}\right)$ by induction on $n$. Fix a recursive list $\left\langle t_{n} \mid n \in \omega\right\rangle$ of terms so that $\left\langle t_{n}\left(k_{1}, \ldots, k_{n}\right)\right| n$ even $\rangle$ enumerates all elements of $L_{k_{\omega}}$.

Let $p_{0}=p_{1}=(\phi,(\phi, \phi))$. Assume that $p_{n}=\left(\bar{s}_{n},\left(p_{n}^{0}, p_{n}^{1}\right)\right)$ has been defined and we wish to define $p_{n+1}=\left(\bar{s}_{n+1},\left(p_{n+1}^{0}, p_{n+1}^{1}\right)\right) \leq p_{n}, n \geq 1$. There are two cases. 
$n$ ODD

We assume inductively (as in Jensen's construction) that $\mathbb{Q} \Vdash \forall u\left(p_{n}^{1}(u)\right.$ is a Jensen-style condition with domain $\left[\omega, k_{1}^{+}\right] \cap L$-Card such that $p_{n}^{1}(u)\left(k_{1}^{+}\right)=$ $(x, \phi)$ where if $p^{*}$ is defined from $k_{2}, \ldots, k_{n}$ as was $p_{n-1}^{1}(u)$ from $k_{1}, \ldots$, $k_{n-1}$, we have $p_{k_{1}^{+}}^{*}=x$.

Now using Lemma 16 choose $\bar{p}_{n+1} \leq p_{n}$ least so that for $\gamma \in L$-Card $\cap\left[\omega, k_{1}\right], \bar{p}_{n+1}$ reduces each $\Delta \in \Sigma_{1}$ Skolem hull $\left(\gamma \cup\left\{k_{1}, \ldots, k_{n}\right\}\right)$ in $L$ below $\gamma$ on the $\mathbb{R}$-component, provided $\Delta$ is predense on the $\mathbb{R}$-component and $\Delta \in L_{k_{1}^{++}}$. Also if $n=1$ require that $\bar{p}_{2}$ reduces each $\Delta \in L_{k_{1}+1}$ below $k_{1}$ on the $\mathbb{R}$-component, provided $\Delta$ is predense on the $\mathbb{R}$-component for the forcing $\hat{\mathscr{P}} \cap L_{k_{1}}$. (The latter is possible using Beller's proof of Lemma 5.3, [BJW].) We also require that $q\left(\bar{p}_{n+1}\right)^{0}=p_{n}^{0}$. Now modify $\bar{p}_{n+1}^{1}=q\left(\bar{p}_{n+1}\right)^{1}$ to obtain $p_{n+1}^{1}$ as follows: $p_{n+1}^{1}(u) \uparrow k_{1}^{+}=\bar{p}_{n+1}^{1}(u) \uparrow k_{1}^{+}, p_{n+1}^{1}(u)\left(k_{1}^{+}\right)=(x, \phi)$ where if $p^{*}$ is defined from $k_{2}, \ldots, k_{n+1}$ as was $p_{n}^{1}(u)$ from $k_{1}, \ldots, k_{n}$ then $p_{k_{1}^{+}}^{*}=x$. Then $p_{n+1}=\left(\bar{s}_{n},\left(p_{n}^{0}, p_{n+1}^{1}\right)\right)=\left(\bar{s}_{n+1},\left(p_{n+1}^{0}, p_{n+1}^{1}\right)\right)$.

\section{$n$ EVEN}

Consider $\Delta=t_{n}\left(k_{1}, \ldots, k_{n}\right)$. If $\Delta$ is predense on the $\mathbb{Q}$-component, $\Delta \in$ $L_{k_{2}^{+}}$then choose $\bar{p}_{n+1} \leq p_{n}$ to be the least condition meeting $\Delta, q\left(\bar{p}_{n+1}\right)^{1}=p_{n}^{1}$.

We would like to include $\bar{p}_{n+1}$ in our generic, but doing so may contradict our desire to build a generic preserved by embeddings derived from shifting indiscernibles. In fact it is to solve this difficulty that we introduced $\mathscr{C}$ as a coding with perfect trees, rather than with finite conditions.

First we may assume that $\bar{p}_{n+1}$ strongly meets $\Delta$ in the sense that it still meets $\Delta$ even after $q\left(\bar{p}_{n+1}\right)^{0}(u)\left(k_{2}\right)\left(i_{1}, \ldots, i_{m}\right) \uparrow k_{1}$ has been constructibly changed for $u \in\left(2^{<\omega}\right)^{<\omega},\left(i_{1}, \ldots, i_{m}\right) \in \operatorname{Dom}\left(q\left(\bar{p}_{n+1}\right)^{0}(u)\left(k_{2}\right)\right)$. This is because the forcing $\mathbb{Q}\left(k_{2}\right)$ obeys $\leq k_{1}^{+}$-distributivity.

Now we would like to alter $q\left(\bar{p}_{n+1}\right)^{0}(u)\left(k_{2}\right)\left(i_{1}, \ldots, i_{m}\right)$ so that it is forced by $\mathbb{Q}^{k_{2}}$ to extend $\underline{G}^{k_{2}}(u)\left(k_{1}\right)\left(\bar{i}_{1}, \ldots, \bar{i}_{m}\right)$ whenever $\left(\bar{i}_{1}, \ldots, \bar{i}_{m}\right)$ is defined from $k_{1} \cup\left\{k_{1}, \ldots, k_{l-1}\right\}$ (some $l$ ) just as $\left(i_{1}, \ldots, i_{m}\right)$ is defined from $k_{1} \cup$ $\left\{k_{2}, \ldots, k_{l}\right\}$. Note that if $\left(i_{1}, \ldots, i_{m}\right)$ is definable from $k_{1} \cup\left\{k_{2}, \ldots, k_{l}\right\}$ then since (we are assuming) $\left(i_{1}, \ldots, i_{m}\right) \in \operatorname{Dom}\left(q\left(\bar{p}_{n+1}\right)^{0}(u)\left(k_{2}\right)\right)$ we have that $\left(i_{1}, \ldots, i_{m}\right)$ is definable from $k_{2} \cup\left\{k_{2}, \ldots, k_{n}\right\}$ and hence from $k_{1} \cup$ $\left\{k_{2}, \ldots, k_{n}\right\}$. (No ordinal in $\left[k_{1}, k_{2}\right.$ ) is definable from $k_{2}, \ldots, k_{l}$.) Thus we can assume that $l=n$. 
The problem is that the above change may not be legal for all $u \in\left(2^{<\omega}\right)^{<\omega}$ since it may require $k_{1} \notin I\left(i_{1}, \ldots, i_{m}\right)$ for $u$-bad $\left(i_{1}, \ldots, i_{m}\right), i_{1}=k_{2}$. Thus we proceed as follows: Extend $\bar{s}\left(\bar{p}_{n+1}\right)$ to guarantee that the above changes can be made for $u$ lying on $\bar{s}\left(\bar{p}_{n+1}\right)$ and then change $q\left(\bar{p}_{n+1}\right)^{0}(u)\left(k_{2}\right)\left(i_{1}, \ldots, i_{m}\right)$ accordingly for such $u$, if possible; otherwise (for technical reasons) extend $\bar{s}\left(\bar{p}_{n+1}\right)$ so as to guarantee that length (stem $\left.\left(\bar{s}\left(\bar{p}_{n+1}\right)(u)\right)\right) \geq n$ for all $u \in$ $\left(2^{<\omega}\right)^{<\omega}$ and that $x_{i}$ is not a path through $\bar{s}\left(\bar{p}_{n+1}\right)\left(u_{j}\right)$ for $i, j \leq n$, where $\left\langle x_{i}\right| i\langle\omega\rangle$ is a fixed enumeration of the constructible reals and $\left\langle u_{j}\right| j\langle\omega\rangle$ is a fixed enumeration of $\left(2^{<\omega}\right)^{<\omega}$. We refer to the former case as the good subcase and the latter as the bad subcase.

Finally let $p_{n+1}=\left(\bar{s}_{n+1},\left(p_{n+1}^{0}, p_{n+1}^{1}\right)\right)$ be the result of the above changes. We will show that in fact the bad subcase does not occur (Lemma 17).

This completes the construction of the $p_{n}$ 's. We obtain a procedure by setting $p\left(i_{1}, \ldots, i_{n}\right)=$ that object defined from $\left(i_{1}, \ldots, i_{n}\right)$ as was $p_{n}$ defined above from $k_{1}, \ldots, k_{n}$, provided the construction above does not break down by stage $n$ where $\left(k_{1}, \ldots, k_{n}\right)$ is replaced by $\left(i_{1}, \ldots, i_{n}\right)$. If the construction breaks down when $\left(k_{1}, \ldots, k_{n}\right)$ is replaced by $\left(i_{1}, \ldots, i_{n}\right)$ then set $p\left(i_{1}, \ldots, i_{n}\right)=\phi$. Thus we have defined a total procedure. To see that this procedure is $\Sigma_{1}(L)$ note the following: In the odd case we refer to $k_{1}^{++}$and $\Sigma_{1}$ Skolem hull $\left(\gamma \cup\left\{k_{1}, \ldots, k_{n}\right\}\right)$ in $L$ but both of these are computable in $L_{k_{n+1}}$ as $n+1 \geq 2$, and $\mathbb{Q} \Vdash \varphi$ is definable over $L_{k_{n+1}}$ for sentences $\varphi$ of rank $<k_{n+1}$. In the even case we refer to $k_{2}^{+}$, which is computable in $L_{k_{n+1}}$ since $n+1 \geq 3$. And in all cases predensity of $\Delta \in L_{k_{n+1}}$ on the appropriate component can be tested in $L_{k_{n+1}}$. So all quantifiers in the definition of $p_{n+1}=p\left(k_{1}, \ldots, k_{n+1}\right)$ can be bounded by $L_{k_{n+1}}$.

Assume henceforth that $\hat{\mathscr{P}}$ was defined using a correct guess for a $\Sigma_{1}(L)$ index for $\left(i_{1}, \ldots, i_{n}\right) \longmapsto p\left(i_{1}, \ldots, i_{n}\right)$. This enables us to prove the following key lemma.

Lemma 17. The good subcase occurs in the case $n$ even.

Proof. Suppose not. Then note that the bad subcase ensures that $\left\langle\bar{s}_{n} \mid n<\omega\right\rangle$ "converges" to a sequence of nonconstructible reals $R(u)=$ unique path through all of the $\bar{s}_{n}(u), n<\omega$ (whenever $u$ lies on each $\bar{s}_{n}$ ). Consider $\bar{s}_{n}(\phi)$. We claim that $s \subseteq R(\phi)$ implies that $k_{1} \notin I\left(i_{1}, \ldots, i_{m}\right)$ for $\langle s\rangle-\operatorname{bad}\left(i_{1}, \ldots, i_{m}\right)$, $i_{1}=k_{2}$ which are definable from $k_{1} \cup\left\{k_{2}, \ldots, k_{n}\right\}$. For, otherwise define $\left(j_{1}, \ldots, j_{m}\right)$ from $k_{1} \cup\left\{k_{m+1}, \ldots, k_{m+n-1}\right\}$ just as $\left(i_{1}, \ldots, i_{m}\right)$ is defined from $k_{1} \cup\left\{k_{2}, \ldots, k_{n}\right\}$. We have that $\left(j_{1}, \ldots, j_{m}\right)$ is also $\langle s\rangle$-bad and by indiscernibility $k_{1}, \ldots, k_{m} \in I\left(j_{1}, \ldots, j_{m}\right)$. Thus by Lemma $1(\mathrm{~b}),\left(k_{1}, \ldots, k_{m}\right)$ is also $\langle s\rangle$-bad. But since we are using a correct guess for an index for our $\Sigma_{1}(L)$ procedure, $p\left(k_{1}, \ldots, k_{m}\right)_{0}=\bar{s}_{m}$ and $\langle s\rangle$ lies on $\bar{s}_{m}$, contradiction.

Thus $\bar{s}_{n}(\phi)$ has a nonconstructible path whose finite initial segments $s$ have 
the property that the changes required in the good subcase can be successfully made for $\langle s\rangle$. A constructible tree with a nonconstructible path contains a constructible perfect subtree, so we can thin $\bar{s}_{n}(\phi)$ as required by the good subcase. Similarly we can successively thin $\bar{s}_{n}(u)$ for $u$ lying on $\bar{s}_{n}$ to obtain the desired extension of $\bar{s}\left(\bar{p}_{n+1}\right)$. We can preserve the condition $\bar{s}_{n}(u) \in \mathscr{C}(u)$ by first extending $\bar{s}_{n}$ so that the above thinnings do not increase constructibility rank.

Now define $K=\left\{p \in \hat{\mathscr{P}} \mid p\right.$ is compatible with each $\left.p(\vec{k}), \vec{k} \in I^{<\omega}\right\}$.

Lemma 18. $K$ is $\hat{\mathscr{P}}$-generic.

Proof. We show that $\hat{G}=\left\{\left(\bar{s}(p), q(p)^{0}\right) \mid p \in K\right\}, \hat{H}=\left\{\left(\bar{s}(p), q(p)^{1}\right) \mid p \in K\right\}$ are $\hat{P}$-generic on the $\mathbb{Q}, \mathbb{R}$-components, respectively.

Notation. $p\left(l_{1}, \ldots, l_{n}\right)^{i}$ denotes $q\left(p\left(l_{1}, \ldots, l_{n}\right)\right)^{i}$.

First consider $\hat{H}$. The inductive requirement in the case $n$ odd guarantees that $\hat{H}$ is "determined" by a compatible class of conditions: indeed for any $\left(l_{1}, \ldots, l_{n}\right) \in I^{n}, p\left(l_{1}, \ldots, l_{n}\right)^{1}$ is forced to agree with $p_{n}^{1}=p\left(k_{1}, \ldots, k_{n}\right)^{1}$ below $k_{1}, p\left(l_{1}, \ldots, l_{n}\right)^{1}(u)_{k_{1}}=p_{n}^{1}(u)_{k_{1}}, p\left(l_{1}, \ldots, l_{n}\right)^{1}(u)_{k_{1}^{+}}=p_{n}^{1}(u)_{k_{1}^{+}}$for all $u$. We may have that $p\left(l_{1}, \ldots, l_{n}\right)^{1}(u), p\left(k_{1}, \ldots, k_{n}\right)^{1}(u)$ impose different restraints, however, at $k_{1}, k_{1}^{+}$as the former must have $\phi$ restraint at $k_{1}$ (if $l_{1}>k_{1}$ ) and the latter must have $\phi$ restraint at $k_{1}^{+}$. Nevertheless we see that they are compatible. Now suppose $\Delta \in L_{\gamma^{++}}$is predense on the $\mathbb{R}$-component, $K$ does not meet $\Delta$ and $\gamma \in L$-Card is least with this property for some $\Delta$. If $\gamma \notin I$ then $\Delta \in \Sigma_{1}$ Skolem hull $\left(\gamma \cup\left\{l_{1}, \ldots, l_{n}\right\}\right)$ in $L$ for some large $l_{1}, \ldots, l_{n} \in I$ and therefore $p\left(l_{1}, \ldots, l_{n}\right)^{1}$ reduces $\Delta$, contradicting the leastness of $\gamma$, unless $\gamma=\omega$. (If $\gamma=\omega$ then $\Delta$ is met due to the $\hat{\mathscr{P}}_{\text {-genericity }}$ of $\hat{G}$ on the $\mathbb{Q}$-component.) Otherwise $p\left(\gamma, l_{2}, \ldots, l_{n}\right)^{1}$ reduces $\Delta$ for some $\left(l_{2}, \ldots, l_{n}\right) \in I$, again contradicting the leastness of $\gamma$. Genericity on the $\mathbb{R}$ component for $L$-definable classes now follows from our choice of $\bar{p}_{2}$.

Now consider $\hat{G}$. Suppose $u \in\left(2^{<\omega}\right)^{<\omega}$; we claim that $p\left(k_{1}, \ldots, k_{n}\right)^{0}(u)$, $p\left(k_{2}, \ldots, k_{n+1}\right)^{0}(u)$ are compatible. Indeed, the only possible conflict would be between $p\left(k_{1}, \ldots, k_{n}\right)^{0}(u)\left(k_{2}\right)$ and $p\left(k_{2}, \ldots, k_{n+1}\right)^{0}(u)\left(k_{2}\right)$ and these are compatible by choice of $\bar{p}_{n+1}^{0}$ (see Lemma 17). Also $p\left(k_{1}, \ldots, k_{n}\right)^{0}(u)$, $p\left(k_{1}, k_{3}, \ldots, k_{n+1}\right)^{0}(u)$ are clearly compatible, as well as $p\left(k_{1}, \ldots, k_{n}\right)^{0}(u)$, $p\left(k_{3}, \ldots, k_{n+2}\right)^{0}(u)$. It now follows that $p(\vec{k})^{0}, p(\vec{l})^{0}$ are compatible in $\mathbb{Q}$ for all $\vec{k}, \vec{l} \in I^{<\omega}$. Suppose $\Delta \in L_{l_{2}^{+}}$is predense on $\mathbb{Q}$-coordinate, where $\left(l_{1}, l_{2}, \ldots, l_{n+1}\right) \in I^{n+1}$ and $\Delta=t_{n}\left(l_{1}, \ldots, l_{n}\right)$. Then $p\left(l_{1}, \ldots, l_{n+1}\right)$ meets $\Delta$, since $p\left(k_{1}, \ldots, k_{n+1}\right)$ meets $t_{n}\left(k_{1}, \ldots, k_{n}\right)$. But now suppose $\Delta=$ $t_{n}\left(l_{1}, \ldots, l_{j}, l_{j+1}, \ldots, l_{n}\right)$ where $\Delta \in L_{l_{j+1}^{+}}$is predense on the $\mathbb{Q}$-component. Then we see that for some $\alpha<l_{j+1}^{+}, \Delta^{*}=\left(\right.$ all $p \in \hat{\mathscr{P}} \cap L_{\alpha}$ reducing each $\Delta$ in 
$\left\{t_{n}\left(\bar{l}_{1}, \ldots, \bar{l}_{j_{d}}, l_{j}, \ldots, l_{n}\right) \mid t_{n}\left(\bar{l}_{1}, \ldots, \bar{l}_{j-1}, l_{j}, \ldots, l_{n}\right)\right.$ is predense on the $\mathbb{Q}$ component and belongs to $\left.L_{l_{j+1}^{+}}\right\}$below $l_{j+1}$ ) is predense on the $\mathbb{Q}$-component, belongs to $L_{l_{j+1}^{+}}$, is definable as $t_{m}\left(l_{j}, l_{j+1}, \ldots, l_{j+m-1}\right)$ for some $m$ and hence is met by $K$. (We have used the $\leq l_{j}$-distributivity of $\mathbb{Q}_{l_{j+1}}$.) Thus if $l_{j+1}$ were chosen to be least so that some $\Delta \in L_{l_{i+1}^{+}}$is predense on the $\mathbb{Q}$-component but not met by $K$, we get a contradiction. So $\hat{G}$ is $\hat{\mathscr{P}}$-generic on the $\mathbb{Q}$ component, as any $L$-definable class predense on the $\mathbb{Q}$-component contains a set which is predense on the $\mathbb{Q}$-component.

Finally we must see that $K$ is compatible. Suppose $\left(\bar{s},\left(p^{0}, p^{1}\right)\right) \in K$. Then $\bar{s}_{n}(u) \leq s_{n}(u)$ for $u$ lying on $\bar{s}_{n}$ (some $n$ ) by genericity on the $\mathbb{Q}$-component. And for $u$ lying on $\bar{s}_{n}$ we must have $p(\vec{l})^{0}(u) \leq p^{0}(u)$ for some $\vec{l} \in I^{<\omega}$ since genericity on the $\mathbb{Q}$-component implies that $\left\{r \in \mathbb{Q}(u) \mid p(\vec{l})^{0}(u) \leq r\right.$ for some $\left.\vec{l} \in I^{<\omega}\right\}$ is $\mathbb{Q}(u)$-generic. And for $u$ lying on $\bar{s}_{n}$, for some $\vec{l}, \vec{l}_{1}, \ldots, \vec{l}_{n} \in I^{<\omega}$ we have $p(\vec{l})^{0}[u] \Vdash p^{1}(u) \geq$ greatest lower bound $\left\langle p\left(\vec{l}_{i}\right)^{1}(u) \mid 1 \leq i \leq n\right\rangle$, since genericity on the $\mathbb{Q}, \mathbb{R}$-component guarantees that $\left\{\left(r^{0}, r^{1}\right) \mid p(\vec{l})^{0}(u) \leq r^{0}\right.$ for some $\vec{l} \in I^{<\omega}$, g.l.b. $\left\langle p\left(\vec{l}_{i}\right)^{1}(u)\right| 1 \leq i \leq$ $n\rangle \leq r^{1}$ for some $\left.\vec{l}_{1}, \ldots, \vec{l}_{n} \in I^{<\omega}\right\}$ is $\mathbb{Q}(u) * \mathbb{R}^{u}$-generic. Moreover any two conditions obeying all of the above must be compatible with each other via a condition obeying all of the above. Thus $K$ consists exactly of the conditions obeying the above properties and hence $K$ is compatible. By Lemma 15 we are done.

Theorem 1. There is a $\hat{\mathscr{P}}$-generic class $K$. If $R=\left\{u \in\left(2^{<\omega}\right)^{<\omega} \mid u\right.$ lies on $\bar{s}(p)$ for each $p \in K\}$ then:

(a) $0<_{L} R<_{L} 0^{\#}$ and $L[R], L$ have the same cardinals.

(b) $R$ is the unique solution to a $\Pi_{2}^{1}$ formula.

Proof. $R$ preserves cardinals since $K$ does. The $\Pi_{2}^{1}$ formula says: $\forall \alpha\left(L_{\alpha}[R] \vDash\right.$ $Z F_{10} \longrightarrow$ For all $u$ in $R$, each $u$-bad $\left(i_{1}, \ldots, i_{m}\right)$ has been killed). $R$ obeys this by Lemma 3(b). Another solution would entail the existence of a CUB subset of true $\omega_{1}$, disjoint from $I$.

Theorem 2. There is an L-definable forcing $\mathscr{P}$ such that:

(a) $\mathscr{P} \Vdash$ Card $=L$-Card and $V=L[R]$ for some real $R \notin L$

(b) There is a unique $\mathscr{P}$-generic, which is definable over $L\left[0^{\#}\right]$.

\section{Countable $\Pi_{2}^{1}$ sets}

The following result answers a question of Kechris.

Theorem 3. There is a nonempty countable $\Pi_{2}^{1}$ set $X$ such that $R \in X \longrightarrow R$ is not a $\Pi_{2}^{1}$-singleton. 
Proof. Modify $\mathscr{P}$ to a forcing $\tilde{\mathscr{P}}$ where we now have a $\Sigma_{1}(L)$ procedure that produces an $\omega$-sequence of conditions $\left\langle p\left(m, i_{1}, \ldots, i_{n}\right) \mid m<\omega\right\rangle$ at each guess $\left(i_{1}, \ldots, i_{n}\right)$. Now for each $s \in\left(2^{<\omega}\right)^{<\omega}$ and each $m<\omega$ we have a Backwards Easton iteration $\mathbb{Q}(m, s)$ for the purpose of killing $\left(i_{1}, \ldots, i_{n}\right)$ when $s$ is incompatible with $p\left(m, i_{1}, \ldots, i_{n}\right)_{0}$. Then $\mathbb{R}(m, s)$ codes the $\mathbb{Q}(m, s)$ generic by $X(m, s)$, a subset of $\omega_{1}^{L}$, and $\mathscr{C}(m, s)$ codes $X(m, s)$ by a real using perfect trees. The procedure $\left(i_{1}, \ldots, i_{n}\right) \longmapsto p\left(m, i_{1}, \ldots, i_{n}\right)$ is

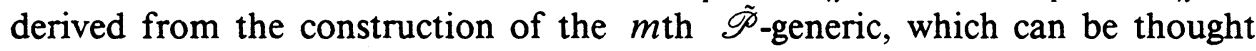
of as a real $R(m)$ where $m$ is an index $m<\omega$ and $R(m)=\{s \mid s$ lies on $p\left(m, i_{1}, \ldots, i_{n}\right)_{0}$ for all $\left.i_{1}, \ldots, i_{n} \in I\right\}$ is designed to kill a guess $\left(i_{1}, \ldots, i_{n}\right)$ whenever $p\left(m^{\prime}, i_{1}, \ldots, i_{n}\right)$ is incompatible with $R(m)$ for all $m^{\prime}$.

The construction of a $\mathscr{P}$-generic proceeds as follows: First choose a condition $p_{0} \in \tilde{\mathscr{P}}$ which reduces each $\Pi_{2}^{1}$ sentence below $\omega$ (on both the $\mathbb{Q}$ and $\mathbb{R}$ components). Thus to decide a $\Pi_{2}^{1}$ sentence we need only extend $\bar{s}\left(p_{0}\right)$. Using fusion we can in fact arrange that this extension can be required to be of the form $\bar{s}\left(p_{0}\right)_{s}$ for some $s \in\left(2^{<\omega}\right)^{<\omega}$ (where $\bar{s}\left(p_{0}\right)_{s}$ is characterized by: $t$ lies on $\bar{s}\left(p_{0}\right)_{s}$ iff $t$ lies on $\bar{s}\left(p_{0}\right)$ and $t$ is compatible with $\left.s\right)$. Now let $\left\langle s_{m} \mid m<\omega\right\rangle$ list all elements of $\left(2^{<\omega}\right)^{<\omega}$ lying on $\bar{s}\left(p_{0}\right)$ and define $R(m)$ as in the $\hat{\mathscr{P}}_{\text {-generic }}$ construction but arrange that the generic $G(m)$ determined by $R(m)$ contains $p_{0}$ and that $R(m)$ extends $s_{m}$. We can also arrange at the first stage of the construction that the reals $R(m), \quad m<\omega$ code different information below $\omega_{1}^{L}$ by making the $X(m, \phi), m<\omega$ distinct.

Let $X=\{R(m) \mid m<\omega\}$. Then $X$ is $\Pi_{2}^{1}$ as $R \in X$ iff $R$ kills each $\left(i_{1}, \ldots, i_{n}\right)$ such that for all $m, p\left(m, i_{1}, \ldots, i_{n}\right)$ is incompatible with $R$ (iff this is true in all $Z F_{10}$ models $\left.L_{\alpha}[R]\right)$. Finally if $R(m)$ satisfies a $\Pi_{2}^{1}$ formula $\phi$ then for some $s$ lying on $R(m)$, so does $R(n)$ whenever $s_{n}$ extends $s$. This proves that $R(m)$ is not a $\Pi_{2}^{1}$-singleton.

On the other hand, [KW] shows that any nonempty countable $\Pi_{2}^{1}$ set $X$ must contain a real $R$ characterized by $L[R] \vDash \phi\left(R, \aleph_{1}, \ldots, \aleph_{n}\right)$, for some $\phi$ and $n<\omega$. Their result is best possible:

Theorem 4. For each $n$ there is a nonempty countable $\Pi_{2}^{1}$ set $X_{n}$ such that $R \in X_{n} \longrightarrow R$ is not characterized by $L[R] \vDash \phi\left(R, \aleph_{1}, \ldots, \aleph_{n}\right)$ for any $\phi$.

Proof. As in the proof of Theorem 3, except choose $p_{0}$ to be the least condition reducing sentences $L[R] \vDash \phi\left(R, i_{1}, \ldots, i_{n}\right)$, when defining $p\left(m, i_{1}, \ldots, i_{n}\right)$. (The construction of $R(m)$ begins with $p\left(m, i_{1}, \ldots, i_{n}\right), i_{1}, \ldots, i_{n} \in I$.) Then any sentence of the form $L[R] \vDash \phi\left(R, \aleph_{1}, \ldots, \aleph_{n}\right)$ true of $R(m)$ is satisfied by other $R(n)$ 's, as $\aleph_{1}, \ldots, \aleph_{n}$ are indiscernibles for any real.

Theorem 5. There is a real $R$ such that:

(a) For some $\phi, R$ is characterized by $L[R] \vDash \phi\left(R, \aleph_{1}\right)$.

(b) $R$ does not belong to any countable $\Pi_{2}^{1}$ set. 
Proof. Build $R$ as in the $\Pi_{2}^{1}$-singleton construction but only kill guesses $\left(i_{1}, \ldots, i_{n}\right)$ where $i_{1} \geq \aleph_{1}$ (and only apply David's trick at or above $\aleph_{1}$ ). Clearly (a) holds. For each $s \in\left(2^{<\omega}\right)^{<\omega}$ the forcing $\mathbb{R}_{\aleph_{1}}^{s}=\left\{p\left|\left[\aleph_{1}, \infty\right)\right| p \in \mathbb{R}^{s}\right\}$ produces $Y(s) \subseteq \aleph_{1}^{+}$(in the sense of $L$ ) so that $Y(s)$ codes $G(s)=$ generic for $\mathbb{Q}(s)$. Then $R$ is generic over $L_{\aleph_{1}}$ for the forcing $\mathscr{\mathscr { P }}$ that adds a real $S$ such that $s$ lies on $S$ iff $S$ codes $Y(s)$. Now if $L[R] \vDash \psi(R)$ where $\psi$ has only countable ordinals as parameters then this is true in $L_{\aleph_{1}}[R]$ and is forced by some condition in $\overline{\mathscr{P}}$ satisfied by $R$. We can build a perfect set of $\overline{\mathscr{P}}$-generics below this condition, yielding uncountably many $S$ such that $L[S] \vDash \psi(S)$.

Remark. A similar result can be established with $\phi\left(R, \aleph_{1}\right), \Pi_{2}^{1}$ replaced by $\phi\left(R, \aleph_{1}, \ldots, \aleph_{n+1}\right), X$ of the form $\left\{S \mid L[S] \vDash \psi\left(S, \aleph_{1}, \ldots, \aleph_{n}\right)\right\}$

Theorem 6. There exists a sequence $\left\langle\left(R_{0}(n), R_{1}(n)\right) \mid n<\omega\right\rangle$ of pairs of reals such that:

(a) $R \leq_{L} R_{0}(n), \quad R \leq_{L} R_{1}(n) \longrightarrow R={ }_{L} 0$.

(b) The predicate " $R=R_{i}(n)$ " is $\Pi_{2}^{1}$ in $R, i, n$.

(c) $n \in 0^{\#} \longleftrightarrow 0 \in R_{0}(n) \longleftrightarrow 0 \in R_{1}(n)$.

Proof. Given $n$, construct a pair of $\Pi_{2}^{1}$-singletons $R_{0}(n), R_{1}(n)$ obeying (a) via the forcing $\hat{\mathscr{P}}(n)$, a modification of $\hat{\mathscr{P}}$. The construction of $R_{0}(n), R_{1}(n)$ places 0 into these reals exactly if $n$ belongs to $0^{\#}$, codes $n$ into the next $n+1$ places of $R_{0}(n), R_{1}(n)$ by putting $k$ into $R_{0}(n), R_{1}(n)$ for $0<k \leq n+1$ iff $k=n+1$, and then builds $R_{0}(n), R_{1}(n)$ to be $\hat{\mathscr{P}}(n)$-generic using the enumeration of constructible reduction procedures in $\omega$ steps given by $0^{\#}$ to guarantee (a). Of course $R_{i}(n)$ codes information to kill any guess at indiscernibles which (via a uniformly defined good indes $f(n)$ ) produces information about the $\hat{\mathscr{P}}(n)$-generic contradicting $R_{i}(n)$. So $R_{i}(n)$ is a $\Pi_{2}^{1}$-singleton, uniformly in $i, n$.

Corollary 7. Consider $X=\left\{R \mid R\right.$ is a $\Pi_{2}^{1}$-singleton and every $\Pi_{2}^{1}$-singleton is $\leq_{L}$-comparable with $\left.R\right\}$. Then the $L$-degrees of elements of $X$ are well ordered, with successor given by \#.

Proof. Suppose that for each $n$, either $R_{0}(n) \leq_{L} R$ or $R_{1}(n) \leq_{L} R$. Then $n \in 0^{\#} \longleftrightarrow L[R] \vDash \exists s \exists i\left[S=R_{i}(n)\right.$ and $\left.0 \in S\right]$. So $0^{\#} \leq_{L} R$. If $R \leq_{L} R_{0}(n)$, $R \leq_{L} R_{1}(n)$ for some $n$ then $R={ }_{L} 0$. Thus $L$-degree $\left(0^{\#}\right)$ is the successor to 0 in the $L$-degrees represented by $X$. By relativization we get that the successor to any $L$-degree represented by an element of $X$ is given by \#.

The $L$-degrees of elements of $X$ are obviously linearly ordered. But an infinite descending chain would give a sequence $\left\langle R_{n} \mid n<\omega\right\rangle, R_{n+1}^{\#} \leq_{L} R_{n}$ leading to an infinite descending sequence of ordinals $\left\langle\omega_{1}^{L\left[R_{n}\right]} \mid n<\omega\right\rangle$, contradiction. 


\section{SOME OPEN QUESTIONS}

(1) Is there a $\Pi_{2}^{1}$-singleton of minimal $L$-degree?

(2) Is there a ZFC-provable $\Pi_{2}^{1}$-singleton $R, 0<_{L} R<_{L} 0^{\#}$ ? ( $R$ is a ZFCprovable $\Pi_{2}^{1}$-singleton if $Z F C \vdash \phi$ has at most one solution, where $\phi$ is a $\Pi_{2}^{1}$ formula characterizing $R$.) [S] has obtained a partial result in this direction.

(3) Consider $X$ as in Corollary 7. Is every $\Pi_{2}^{1}$-singleton constructible from an element of $X$ ? Are the $L$-degrees of sharps of $\Pi_{2}^{1}$-singletons well ordered?

\section{REFERENCES}

[BJW] A. Beller, R. Jensen, and P. Welch, Coding the universe, Cambridge Univ. Press, Cambridge, 1982.

[D] R. David, $A$ very absolute $\Pi_{2}^{1}$-singleton, Ann. Pure and Appl. Logic 23 (1982), 101-120.

[F1] S. Friedman, An Immune partition of the ordinals, Recursion Theory Week, Lecture Notes in Math., vol. 1144, Springer-Verlag, New York, 1986, pp. 141-147.

[F2] _ Minimal coding, Ann. Pure and Appl. Logic 41 (1989), 233-297.

[JS] R. Jensen and R. Solovay, Some applications of almost disjoint sets, Mathematical Logic and the Foundations of Set Theory, North-Holland, 1968, pp. 84-104.

[KW] A. Kechris and W. H. Woodin, On thin $\Pi_{2}^{1}$ sets, handwritten note, 1983.

[S] M. Stanley, An absolute $\Pi_{2}^{1}$-singleton (to appear).

ABSTRACT. The real $0^{\#}=\operatorname{Thy}\left\langle L, \varepsilon, \aleph_{1}, \aleph_{2}, \ldots\right\rangle$ is a natural example of a nonconstructible definable real. Moreover $0^{\#}$ has a definition that is absolute: for some formula $\phi(x), 0^{\#}$ is the unique real $R$ such that $L[R] \vDash \phi(R)$. Solovay conjectured that there is a real $R$ such that $0<_{L} R<_{L} 0^{\#}$ and $R$ also has such an absolute definition. We prove his conjecture by constructing a $\Pi_{2}^{1}$-singleton $R, \quad 0<_{L} R<_{L} 0^{\#}$. A variant of our construction produces a countable nonempty $\Pi_{2}^{1}$ set of reals not containing a $\Pi_{2}^{1}$-singleton. The latter result answers a question of Kechris.

Department of Mathematics, Massachusetts institute of Technology, Cambridge, MASSACHUSETTS 02139 